Research Article

\title{
On Some Coincidence Best Proximity Point Results
}

\author{
Naeem Saleem $\mathbb{D}^{1},{ }^{1}$ Haroon Ahmad, ${ }^{1}$ Hassen Aydi $\mathbb{D}^{2,3,4}$ and Yaé Ulrich Gaba $\mathbb{D}^{4,5}$ \\ ${ }^{1}$ Department of Mathematics, University of Management and Technology, Lahore, Pakistan \\ ${ }^{2}$ Université de Sousse, Institut Supérieur d'Informatique et des Techniques de Communication, H. Sousse 4000, Tunisia \\ ${ }^{3}$ China Medical University Hospital, China Medical University, Taichung 40402, Taiwan \\ ${ }^{4}$ Department of Mathematics and Applied Mathematics, Sefako Makgatho Health Sciences University, P.O. Box 60, \\ Ga-Rankuwa 0208, South Africa \\ ${ }^{5}$ Institut de Mathématiques et de Sciences Physiques (IMSP/UAC), \\ Laboratoire de Topologie Fondamentale, Computationnelle et leurs Applications (Lab-ToFoCApp), Porto-Novo BP 613, Benin
}

Correspondence should be addressed to Hassen Aydi; hassen.aydi@isima.rnu.tn and Yaé Ulrich Gaba; yaeulrich.gaba@gmail.com

Received 25 September 2021; Accepted 23 November 2021; Published 22 December 2021

Academic Editor: Efthymios G. Tsionas

Copyright (C) 2021 Naeem Saleem et al. This is an open access article distributed under the Creative Commons Attribution License, which permits unrestricted use, distribution, and reproduction in any medium, provided the original work is properly cited.

In this paper, we discuss some (coincidence) best proximity point results for generalized proximal contractions and $\lambda-\mu$-proximal Geraghty contractions in controlled metric type spaces. To clarify our study, various examples are given and some conclusions are drawn.

\section{Introduction and Preliminaries}

To solve the equation $\check{T} p=p(\check{T}$ is a mapping defined on a subset of a metric space, a simplified linear space, or a topological vector space), fixed point theory is an important tool. A nonself-mapping $\check{T}: J \longrightarrow K$ may not have a fixed point. From this perspective, the best approximation theorem and the best proximity point are relevant. A classical best approximation theorem was due to Fan [1], i.e., if $J$ is a nonempty compact convex subset of a Hausdorff locally convex topological vector space $X$ with a seminorm $p$ and $\check{T}: J \longrightarrow X$ is a continuous mapping, then there is an element $p$ in $J$ satisfying the condition that $\Psi(p, \breve{T} p)=\Psi(\breve{T} p, J)$. Many subsequent extensions and variations of Fan's theorem have occurred, including references $[2,3]$.

However, even though the best approximation theorems provide an approximate solution to the equation $\breve{T} p=p$, they do not provide an ideal approximate solution. Moreover, the theorem of the best proximity point specifies adequate criteria for the presence of an element $p$ to reduce the error $\Psi(p, \check{T} p)$. For a nonself-mapping $\check{T}: J \longrightarrow K$, $\Psi(p, \check{T} p)$ is at least $\Psi(J, K)$ for all $p$ in $J$, then the best proximity point theorem establishes a globally optimal solution of error $\Psi(p, \breve{T} p)$ by constraining an approximate solution $p$ of the equation $\check{T} p=p$ to the condition that $\Psi(p, \check{T} p)=\Psi(J, K)$. Such an ideal approximate solution $\check{T} p=p$ is the best proximity point of the nonself-mapping $\check{T}: J \longrightarrow K$. For sure, the best proximity point hypotheses are a logical augmentation of fixed point hypotheses, on the grounds that the best proximity point is a fixed point in the light of self-mappings.

The best proximity point hypotheses have been demonstrated in [4]. Anuradha and Veeramani have tested the proximal pointwise contractions for the presence of a best proximity point [2]. Generally, several best proximity point theorems were analyzed for multiple variants of contractions in [5-14]. A best proximity point theorem for contraction mappings was presented in [15]. Some interesting common best proximity theorems have been discussed in $[7,15]$.

Nadler [16] was the first who generalized the Banach contraction principle for multivaluated mappings. Later, several works appeared in this direction. For more details, see $[17-20]$. The best proximity point hypotheses for different sorts of multivalued mappings have likewise been obtained in [21, 22].

Recently, the authors in [23] introduced a controlled metric type space in which the function of extended $b$-metric spaces was substituted by a function $\alpha(p, q)$ depending on 
the parameters of the left-hand side of the triangular inequality. The primary goal of this article is to include the best proximity point theorems for generalized and modified proximal contractions in the context of complete controlled type metric spaces, thus providing an optimal approximate solution to the equation $\breve{T} p=p$. It is acknowledged that the previous best proximity point theorems include the wellknown Banach contraction principle and some of its generalizations.

First, we state the following useful definitions in the sequel.

Definition 1 (see [6]). Let $(X, \Psi)$ be a metric space having a pair of nonempty subsets $(J, K)$ such that $J_{0}$ is nonempty. The pair $(J, K)$ has the $P$-property if and only if

$$
\left.\begin{array}{l}
\Psi\left(p_{1}, q_{1}\right)=\Psi(J, K) \\
\Psi\left(p_{2}, q_{2}\right)=\Psi(J, K)
\end{array}\right\}, \quad \text { implies } \Psi\left(p_{1}, p_{2}\right)=\Psi\left(q_{1}, q_{2}\right),
$$

where $p_{1}, p_{2} \in J_{0}$ and $q_{1}, q_{2} \in K_{0}$.

Definition 2 (see [24]). Let $(X, \Psi)$ be a metric space having a pair of nonempty subsets $J$ and $K$. Let $\check{T}: J \longrightarrow K$ and $\mu: J \times J \longrightarrow[0, \infty)$. The mapping $\check{T}$ is said to be $\mu$-proximal admissible if

$$
\left.\begin{array}{l}
\mu\left(p_{1}, p_{2}\right) \geq 1 \\
\Psi\left(u_{1}, \check{T} p_{1}\right)=\Psi(J, K) \\
\Psi\left(u_{2}, \check{T} p_{2}\right)=\Psi(J, K)
\end{array}\right\}, \quad \text { implies } \mu\left(u_{1}, u_{2}\right) \geq 1,
$$

for all $p_{1}, p_{2}, u_{1}, u_{2} \in J$.

Definition 3 (see [25]). Let $\mathscr{B}(X)$ represent the closed and bounded subsets of $X$. Let $H$ be the Pompeiu-Hausdroff metric induced by metric $\Psi$ defined by

$$
H(J, K)=\max \left\{\sup _{a \in J} \mathscr{D}(a, K), \sup _{b \in K} \mathscr{D}(b, J)\right\},
$$

for $J, K \subseteq \mathscr{B}(X)$, where

$$
\mathscr{D}(a, K)=\inf \{\Psi(a, b): b \in K\} .
$$

Definition 4 (see [23]). Let $X$ be a nonempty set, and consider $\alpha: X \times X \longrightarrow[1, \infty)$ as a function. Let $\Psi: X \times X \longrightarrow[0, \infty)$ satisfying

(1) $\Psi\left(p_{1}, p_{2}\right)=0$ if and only if $p_{1}=p_{2}$

(2) $\Psi\left(p_{1}, p_{2}\right)=\Psi\left(p_{2}, p_{1}\right)$

(3) $\Psi\left(p_{1}, p_{2}\right) \leq \alpha\left(p_{1}, p_{3}\right) \Psi\left(p_{1}, p_{3}\right)+\alpha\left(p_{3}, p_{2}\right) \Psi\left(p_{3}, p_{2}\right)$, for all $p_{1}, p_{2}, p_{3} \in X$, then $(X, \Psi)$ is called a controlled metric type space

From now on, $(X, \Psi)$ is a controlled metric type space.

Definition 5 (see [23]). A sequence $\left\{p_{n}\right\}$ in a controlled metric type space $(X, \Psi)$ converges to some $p$ in $X$ if for each positive $\varepsilon$, there is some positive $N_{\varepsilon}$ such that $\Psi\left(p_{n}, p\right)<\varepsilon$ for each $n \geq N_{\varepsilon}$. It can be written as

$$
\lim _{n \longrightarrow \infty} p_{n}=p \text {. }
$$

Definition 6 (see [23]). The sequence $\left\{p_{n}\right\}$ in a controlled metric type space $(X, \Psi)$ is said to be a Cauchy sequence, if for every $\varepsilon>0, \Psi\left(p_{n}, p_{m}\right)<\varepsilon$ for all $m, n \geq N_{\varepsilon}$, where $N_{\varepsilon} \in \mathbb{N}$.

Definition 7 (see [23]). A controlled metric type space $(X, \Psi)$ is said to be complete if every Cauchy sequence is convergent in $X$.

Definition 8 (see [23]). Let $p \in X$ and $\varepsilon>0$.

(1) The open ball $K(p, \varepsilon)$ is defined as follows:

$$
K(p, \varepsilon)=\{q \in X, \Psi(p, q)<\varepsilon\} .
$$

(2) The mapping $\check{T}: X \longrightarrow X$ is said continuous at $p \in X$ if for all $\varepsilon>0$, there exists $\delta>0$ such that

$$
\check{T}(K(p, \delta)) \subseteq K(\check{T} p, \varepsilon) .
$$

Clearly, if $\check{T}$ is continuous at $p$ in the controlled metric type space $(X, \Psi)$, then $p_{n} \longrightarrow p$ implies that $\check{T} p_{n} \longrightarrow \check{T} p$ as $n \longrightarrow \infty$.

Definition 9 (see [26]). Define the function $\mathscr{H}: \mathscr{L}(X) \times \mathscr{L}(X) \longrightarrow[0, \infty]$ by

$$
\mathscr{H}(J, K)= \begin{cases}\max \left\{\sup _{a \in J} \mathscr{D}(a, K), \sup _{b \in K} \mathscr{D}(b, J)\right\}, & \text { if the maximum exists, } \\ \infty, & \text { otherwise, }\end{cases}
$$


for $J, K \subseteq \mathscr{L}(X)$ (it represents the set of closed subsets of $X$ ), where

$$
\mathscr{D}(a, K)=\inf \{\Psi(a, b): b \in K\}, \quad \text { for } K \subset X .
$$

Let $J$ and $K$ be two nonempty subsets of $X$. Define

$$
\begin{aligned}
J_{0} & =\{p \in J: \Psi(p, q)=\Psi(J, K) \text { for some } q \in K\}, \\
K_{0} & =\{q \in K: \Psi(p, q)=\Psi(J, K) \text { for some } p \in J\},
\end{aligned}
$$

where

$$
\Psi(J, K)=\inf \{\Psi(p, q): p \in J, q \in K\} \text { (distance of a set } J \text { to a set } K \text { ), }
$$

and we will denote

$$
\mathscr{D}^{*}(p, q)=\Psi(p, q)-\Psi(J, K), \quad \text { for all } p \in J, q \in K .
$$

Theorem 1 (see [26]). The function $\mathscr{H}: \mathscr{L}(X) \times \mathscr{L}(X)$ $\longrightarrow[0, \infty]$ is a generalized Pompeiu-Hausdroff controlled metric space on $\mathscr{L}(X)$.

Remark 1 (see [26]). Let $(\mathscr{L}(X), H)$ be a generalized Pompeiu-Hausdroff-controlled metric type space. Then, the following assertions hold (for all bounded and closed subsets $J, K, C$, and $D$ of $X$ ):

(1) $H(C, D)=0$ is equivalent to $C=D$

(2) $H(C, D)=H(D, C)$

(3) $H(J, C) \leq \operatorname{maxsup}_{a \in J} \alpha(a, b), \alpha(b, J) H(J, K)+$ $\max \alpha(b, C), \sup _{c \in C} \alpha(c, b), H(K, C)$

Theorem 2 (see [26]). If $(X, \Psi)$ is a complete controlled metric space with $\lim _{n, m \rightarrow \infty} \alpha\left(p_{n}, p_{m}\right) k<1$, for all $p_{n}, p_{m}$ $\in X$, where $k \geq 1$, then $(\mathscr{L}(X), \mathscr{H})$ is complete.

\section{Coincidence Best Proximity Points for Generalized Proximal Contractions}

In this section, we will discuss some best proximity point theorems using the multivalued concept on a controlled metric space $(X, \Psi)$.
From now and onward, $J$ and $K$ are nonempty subsets of a controlled metric type space $(X, \Psi)$ (until otherwise stated). Define $\alpha: X \times X \longrightarrow[1, \infty)$ by $\alpha_{*}(p, J)=$ $\inf \{\alpha(p, a)$, for all $a \in J\}$ and $\alpha_{*}(J, K)=\inf \{\alpha(a, b)$, for all $a \in J$ and $b \in K\}$, where $\alpha: X \times X \longrightarrow[1, \infty)$ and $J$ and $K$ are nonempty subsets of $X$.

Definition 10 (see [26]). A mapping $\check{T}: X \longrightarrow \mathscr{B}(X)$ is continuous in a controlled metric type space $(X, \Psi)$ at $p \in X$ if for all $\varepsilon>0$, there exists $\delta>0$ such that

$$
\check{T}(K(p, \delta)) \subseteq K(\check{T} p, \varepsilon),
$$

where $K(p, \varepsilon)$ is given as

$$
K(p, \varepsilon)=\{q \in X, \Psi(p, q)<\varepsilon\} .
$$

Clearly, if $\check{T}$ is continuous at $p$, then $p_{n} \longrightarrow p$ implies that $\check{T} p_{n} \longrightarrow \check{T} p$ as $n \longrightarrow \infty$.

We introduce the following.

Definition 11. Let $(X, \Psi)$ be a controlled metric type space having two nonempty subsets $J$ and $K$. Let $\check{T}: J \longrightarrow K$ be a mapping. A point $p \in J$ is said to be a best proximity point of the mapping $\breve{T}$ if

$$
\Psi(p, \check{T} p)=\Psi(J, K)
$$

Definition 12. Let $(X, \Psi)$ be a controlled metric type space having two nonempty subsets $J$ and $K$. A nonempty set $J$ is said to be approximately compact with respect to $K$ if every sequence $\left\{p_{n}\right\}$ in $J$ satisfying the condition that $\mathscr{D}\left(q, p_{n}\right) \longrightarrow \mathscr{D}(q, J)$ for some $q$ in $K$ has a convergent subsequence.

Definition 13. Given $\check{T}: J \longrightarrow \mathscr{B}(K)$ and $\breve{g}: J \longrightarrow J$. A pair of mappings $(\breve{g}, \breve{T})$ is said to be a $\beta$-generalized proximal contraction if there exists a real number $\beta \in[0,1)$ such that

$$
\left.\begin{array}{l}
\mathscr{D}\left(\breve{g} u_{1}, \check{T} p_{1}\right)=\Psi(J, K) \\
\mathscr{D}\left(\breve{g} u_{2}, \check{T} p_{2}\right)=\Psi(J, K)
\end{array}\right\}, \quad \text { implies } \mathscr{H}\left(\check{T} u_{1}, \check{T} u_{2}\right) \leq \beta \mathscr{H}\left(\check{T} p_{1}, \check{T} p_{2}\right),
$$


for all $u_{1}, u_{2}, p_{1}$, and $p_{2}$ in $J$.
Definition 14. A mapping $\check{T}: J \longrightarrow \mathscr{B}(K)$ is said to be a $\beta_{\breve{T}^{-}}$ generalized proximal contraction if there exists $\beta \in[0,1)$ such that

$$
\left.\begin{array}{l}
\mathscr{D}\left(u_{1}, \check{T} p_{1}\right)=\Psi(J, K) \\
\mathscr{D}\left(u_{2}, \check{T} p_{2}\right)=\Psi(J, K)
\end{array}\right\}, \quad \text { implies } \mathscr{H}\left(\check{T} u_{1}, \check{T} u_{2}\right) \leq \beta \mathscr{H}\left(\check{T} p_{1}, \check{T} p_{2}\right)
$$

for all $u_{1}, u_{2}, p_{1}$, and $p_{2}$ in $J$.

Note that, if we take $\breve{g}=I_{J}$ (the identity mapping on $J$ ), then every $\beta$-generalized proximal contraction will reduce to a $\beta_{\breve{T}}$-generalized proximal contraction.

Definition 15. Let $(X, \Psi)$ be a controlled metric type space having two nonempty subsets $J$ and $K$. Let $\check{T}: J \longrightarrow K$ and $\breve{g}: J \longrightarrow J$ be mappings. A point $p \in J$ is said to be a coincidence best proximity point of the pair of mappings $(\breve{g}, \breve{T})$ if

$$
\Psi(\breve{g} p, \check{T} p)=\Psi(J, K)
$$

Remark 2. If we take $\breve{g}=I_{J}$ (the identity mapping over $J$ ), then every coincidence best proximity point becomes a best proximity point of the mapping $\check{T}$.

If $J \cap K \neq \varnothing$ or $\Psi(J, K)=0$, then every best proximity point will reduce to a fixed point of the mapping $\check{T}$.

Our first main result is stated as follows:

Theorem 3. Let $(X, \Psi)$ be a controlled metric type space having two nonempty subsets $J$ and $K$. Let $\check{T}: J \longrightarrow \mathscr{B}(K)$ and $\mathrm{g}: J \longrightarrow J$ be one-to-one and continuous mappings. Assume that $K$ is a closed subset and $J$ is approximately compact with respect to $K$ with $\breve{T}\left(J_{0}\right) \subseteq K_{0}$ and $J_{0} \subseteq \breve{g}\left(J_{0}\right)$. Further, assume that the pair $(\breve{g}, \check{T})$ is a $\beta$-generalized proximal contraction such that

$$
\begin{aligned}
& \sup _{m \geq 1} \lim _{i \longrightarrow \infty} \max \left\{\sup _{q_{i} \check{T} p_{i}} \alpha\left(q_{i}, q_{i+1}\right), \alpha\left(q_{i+1}, \check{T} p_{i}\right)\right\} \max \left\{\sup _{\left.q_{i \in \check{T} p_{i}} \alpha\left(q_{i}, q_{m}\right), \alpha\left(q_{m}, \check{T} p_{i}\right)\right\}<\frac{1}{k},}\right. \\
& \mathscr{D}\left(\breve{g} p_{n}, \check{T} p_{n-1}\right)=\Psi(J, K) .
\end{aligned}
$$

Having selected $\left\{p_{n}\right\}$ satisfying the condition, there exists there exists a coincidence best proximity point of the pair $(\breve{g}, \check{T})$.

Proof. Let $p_{0}$ be an arbitrary element in $J_{0}$. Since $\check{T}\left(J_{0}\right)$ is contained in $K_{0}$ and $J_{0}$ is contained in $\breve{g}\left(J_{0}\right)$, there exists an element $p_{1}$ in $J_{0}$ such that

$$
\mathscr{D}\left(\breve{g} p_{1}, \check{T} p_{0}\right)=\Psi(J, K) \text {. }
$$

Again, since $\check{T} p_{1}$ is an element of $\check{T}\left(J_{0}\right)$ which is contained in $K_{0}$ and $J_{0}$ is contained in $\breve{g}\left(J_{0}\right)$, it follows that there is an element $p_{2}$ in $J_{0}$ such that

$$
\mathscr{D}\left(\breve{g} p_{2}, \check{T} p_{1}\right)=\Psi(J, K) .
$$

This process can be continued by selecting $p_{n}$ in $J_{0}$ satisfying the condition as follows: an element $p_{n+1}$ in $J_{0}$ satisfying

$$
\mathscr{D}\left(\breve{g} p_{n+1}, \check{T} p_{n}\right)=\Psi(J, K),
$$

for every integer $n \geq 0$.

Since the pair $(\breve{g}, \check{T})$ is a $\beta$-generalized proximal contraction, by using equations (22) and (23), we obtain

$$
\mathscr{H}\left(\check{T} p_{n+1}, \check{T} p_{n}\right) \leq \beta \mathscr{H}\left(\check{T} p_{n}, \check{T} p_{n-1}\right), \quad \text { for each } n \geq 1 \text {. }
$$

We deduce that

$$
\mathscr{H}\left(\check{T} p_{n+1}, \check{T} p_{n}\right) \leq \beta^{n} \mathscr{H}\left(\check{T} p_{1}, \check{T} p_{0}\right), \quad \text { for each } n \geq 0 \text {. }
$$

Now, we have to prove that $\left\{\check{T} p_{n}\right\}$ is a Cauchy sequence, for all natural numbers $n, m \in \mathbb{N}$ with $n<m$, 


$$
\begin{aligned}
& \mathscr{H}\left(\check{T} p_{n}, \check{T} p_{m}\right) \leq \max \left\{\sup _{q_{n} \in \check{T} p_{n}} \alpha\left(q_{n}, q_{n+1}\right), \alpha\left(q_{n+1}, \check{T} p_{n}\right)\right\} \mathscr{H}\left(\check{T} p_{n}, \check{T} p_{n+1}\right) \\
& +\max \left\{\sup _{q_{n+1} \in \bar{T} p_{n+1}} \alpha\left(q_{n+1}, q_{m}\right), \alpha\left(q_{m}, \check{T} p_{n+1}\right)\right\} \mathscr{H}\left(\check{T} p_{n+1}, \check{T} p_{m}\right) \\
& \leq \max \left\{\sup _{q_{n} \in \check{T} p_{n}} \alpha\left(q_{n}, q_{n+1}\right), \alpha\left(q_{n+1}, \check{T} p_{n}\right)\right\} \mathscr{H}\left(\check{T} p_{n}, \check{T} p_{n+1}\right) \\
& +\max \left\{\sup _{q_{n+1} \in \check{T} p_{n+1}} \alpha\left(q_{n+1}, q_{n+2}\right), \alpha\left(q_{n+2}, \check{T} p_{n+1}\right)\right\} \max \left\{\sup _{q_{n+1} \in \bar{T} p_{n+1}} \alpha\left(q_{n+1}, q_{m}\right), \alpha\left(q_{m}, \check{T} p_{n+1}\right)\right\} \mathscr{H}\left(\check{T} p_{n+1}, \check{T} p_{n+2}\right) \\
& +\max \left\{s \sup _{q_{n+1} \in \check{T} p_{n+1}} \alpha\left(q_{n+1}, q_{m}\right), \alpha\left(q_{m}, \check{T} p_{n+1}\right)\right\} \max \left\{\sup _{q_{n+2} \in \check{T} p_{n+2}} \alpha\left(q_{n+2}, q_{m}\right), \alpha\left(q_{m}, \check{T} p_{n+2}\right)\right\} \mathscr{H}\left(\check{T} p_{n+2}, \check{T} p_{m}\right) \\
& \leq \max \left\{\sup _{q_{n} \in \check{T} p_{n}} \alpha\left(q_{n}, q_{n+1}\right), \alpha\left(q_{n+1}, \check{T} p_{n}\right)\right\} \mathscr{H}\left(\check{T} p_{n}, \check{T} p_{n+1}\right) \\
& +\sum_{i=n+1}^{m-2}\left(\prod_{j=n+1}^{i} \max \left\{\sup _{q_{j} \in \check{T} p_{j}} \alpha\left(q_{j}, q_{m}\right), \alpha\left(q_{m}, \check{T} p_{j}\right)\right\}\right) \max \left\{\sup _{q_{i} \in \check{T} p_{i}} \alpha\left(q_{i}, q_{i+1}\right), \alpha\left(q_{i+1}, \check{T} p_{i}\right)\right\} \mathscr{H}\left(\check{T} p_{i}, \check{T} p_{i+1}\right) \\
& +\prod_{k=n+1}^{m-1} \max \left\{\sup _{q_{n_{k}} \in \check{T} p_{k}} \alpha\left(q_{k}, q_{m}\right), \alpha\left(q_{m}, \check{T} p_{k}\right)\right\} \mathscr{H}\left(\check{T} p_{m-1}, \check{T} p_{m}\right) \\
& \leq \max \left\{\sup _{q_{n} \in \check{T} p_{n}} \alpha\left(q_{n}, q_{n+1}\right), \alpha\left(q_{n+1}, \check{T} p_{n}\right)\right\} \beta^{n} \mathscr{H}\left(\check{T} p_{0}, \check{T} p_{1}\right) \\
& +\sum_{i=n+1}^{m-2}\left(\prod_{j=n+1}^{i} \max \left\{\sup _{q_{j} \in \check{T} p_{j}} \alpha\left(q_{j}, q_{m}\right), \alpha\left(q_{m}, \check{T} p_{j}\right)\right\}\right) \max \left\{\sup _{q_{i} \in \check{T} p_{i}} \alpha\left(q_{i}, q_{i+1}\right), \alpha\left(q_{i+1}, \check{T} p_{i}\right)\right\} \beta^{i} \mathscr{H}\left(\check{T} p_{0}, \check{T} p_{1}\right) \\
& +\prod_{k=n+1}^{m-1} \max \left\{\sup _{q_{n_{k}} \in \check{T} p_{k}} \alpha\left(q_{k}, q_{m}\right), \alpha\left(q_{m}, \check{T} p_{k}\right)\right\} \beta^{m-1} \mathscr{H}\left(\check{T} p_{0}, \check{T} p_{1}\right) \\
& \leq \max \left\{\sup _{q_{n} \in \check{T} p_{n}} \alpha\left(q_{n}, q_{n+1}\right), \alpha\left(q_{n+1}, \check{T} p_{n}\right)\right\} \beta^{n} \mathscr{H}\left(\check{T} p_{0}, \check{T} p_{1}\right) \\
& +\sum_{i=n+1}^{m-2}\left(\prod_{j=n+1}^{i} \max \left\{\sup _{q_{j} \in \check{T} p_{j}} \alpha\left(q_{j}, q_{m}\right), \alpha\left(q_{m}, \check{T} p_{j}\right)\right\}\right) \max \left\{\sup _{q_{i} \in \check{T} p_{i}} \alpha\left(q_{i}, q_{i+1}\right), \alpha\left(q_{i+1}, \check{T} p_{i}\right)\right\} \beta^{i} \mathscr{H}\left(\check{T} p_{0}, \check{T} p_{1}\right) \\
& +\prod_{k=n+1}^{m-1} \max \left\{\sup _{q_{n_{k}} \in \check{T} p_{k}} \alpha\left(q_{k}, q_{m}\right), \alpha\left(q_{m}, \check{T} p_{k}\right)\right\} \max \left\{\sup _{q_{m-1} \in \check{T} p_{m-1}} \alpha\left(q_{m-1}, q_{m}\right), \alpha\left(q_{m}, \check{T} p_{m-1}\right)\right\} \beta^{m-1} \mathscr{H}\left(\check{T} p_{0}, \check{T} p_{1}\right) \\
& =\max \left\{\sup _{q_{n} \in \check{T} p_{n}} \alpha\left(q_{n}, q_{n+1}\right), \alpha\left(q_{n+1}, \check{T} p_{n}\right)\right\} \beta^{n} \mathscr{H}\left(\check{T} p_{0}, \check{T} p_{1}\right)
\end{aligned}
$$




$$
\begin{aligned}
& \quad+\sum_{i=n+1}^{m-2}\left(\prod_{j=n+1}^{i} \max \left\{\sup _{q_{j} \in \check{T} p_{j}} \alpha\left(q_{j}, q_{m}\right), \alpha\left(q_{m}, \check{T} p_{j}\right)\right\}\right) \max \left\{\sup _{q_{i} \in \check{T} p_{i}} \alpha\left(q_{i}, q_{i+1}\right), \alpha\left(q_{i+1}, \check{T} p_{i}\right)\right\} \beta^{i} \mathscr{H}\left(\check{T} p_{0}, \check{T} p_{1}\right) \\
& \leq \max \left\{\sup _{q_{n} \in \check{T} p_{n}} \alpha\left(q_{n}, q_{n+1}\right), \alpha\left(q_{n+1}, \check{T} p_{n}\right)\right\} \beta^{n} \mathscr{H}\left(\check{T} p_{0}, \check{T} p_{1}\right) \\
& +\sum_{i=n+1}^{m-2}\left(\prod_{j=0}^{i} \max \left\{\sup _{q_{j} \in \check{T} p_{j}} \alpha\left(q_{j}, q_{m}\right), \alpha\left(q_{m}, \check{T} p_{j}\right)\right\}\right) \max \left\{\sup _{q_{i} \in \check{T} p_{i}} \alpha\left(q_{i}, q_{i+1}\right), \alpha\left(q_{i+1}, \check{T} p_{i}\right)\right\} \beta^{i} \mathscr{H}\left(\check{T} p_{0}, \check{T} p_{1}\right) .
\end{aligned}
$$

Assume that

$$
S_{m-2}=\sum_{i=n+1}^{m-2}\left(\prod_{j=0}^{i} \max \left\{\sup _{q_{j} \in \check{T} p_{j}} \alpha\left(q_{j}, q_{m}\right), \alpha\left(q_{m}, \check{T} p_{j}\right)\right\}\right) \max \left\{\sup _{q_{i} \in \check{T} p_{i}} \alpha\left(q_{i}, q_{i+1}\right), \alpha\left(q_{i+1}, \check{T} p_{i}\right)\right\} \beta^{i} .
$$

Then, we obtain

$$
\mathscr{H}\left(\check{T} p_{n}, \check{T} p_{m}\right) \leq \mathscr{H}\left(\check{T} p_{0}, \check{T} p_{1}\right)\left[\beta^{n} \max \left\{\sup _{q_{n} \in \check{T} p_{n}} \alpha\left(q_{n}, q_{n+1}\right), \alpha\left(q_{n+1}, \check{T} p_{n}\right)\right\}+\left(S_{m-1}-S_{n}\right)\right] .
$$

Using the ratio test, we have

$$
a_{i}=\prod_{j=0}^{i} \max \left\{\sup _{q_{j} \in \check{T} p_{j}} \alpha\left(q_{j}, q_{m}\right), \alpha\left(q_{m}, \check{T} p_{j}\right)\right\} \max \left\{\sup _{q_{i} \in \check{T} p_{i}} \alpha\left(q_{i}, q_{i+1}\right), \alpha\left(q_{i+1}, \check{T} p_{i}\right)\right\} \beta^{i}
$$

where $\left(a_{i+1} / a_{i}\right)<(1 / k)$. Taking limit as $n, m \longrightarrow \infty$, we obtain

$$
\lim _{n \longrightarrow \infty} \mathscr{H}\left(\check{T} p_{n}, \check{T} p_{m}\right)=0
$$

That is, $\left\{\check{T} p_{n}\right\}$ is a Cauchy sequence in the complete generalized Pompeiu-Hausdroff controlled metric type space $(\mathscr{B}(X), \mathscr{H})$; hence, it converges to some $q$ in $K$ (as the set $K$ is closed). Therefore,

$$
\begin{aligned}
\Psi(q, J) & \leq \Psi\left(q, \breve{g} p_{n}\right) \leq \alpha_{*}\left(q, \breve{T} p_{n-1}\right) \mathscr{D}\left(q, \check{T} p_{n-1}\right)+\alpha_{*}\left(\check{T} p_{n-1}, \breve{g} p_{n}\right) \mathscr{D}\left(\check{T} p_{n-1}, \breve{g} p_{n}\right) \\
& =\alpha_{*}\left(q, \breve{T} p_{n-1}\right) \mathscr{D}\left(q, \breve{T} p_{n-1}\right)+\alpha_{*}\left(\check{T} p_{n-1}, \breve{g} p_{n}\right) \Psi(J, K) .
\end{aligned}
$$
have

Taking $\lim _{n \longrightarrow \infty}$ on both sides of the above inequality, we 


$$
\begin{aligned}
\lim _{n \longrightarrow \infty} \Psi\left(q, \breve{g} p_{n}\right) & \leq \lim _{n \longrightarrow \infty}\left[\alpha_{*}\left(q, \check{T} p_{n-1}\right) \mathscr{D}\left(q, \check{T} p_{n-1}\right)+\alpha_{*}\left(\check{T} p_{n-1}, \breve{g} p_{n}\right) \Psi(J, K)\right] \\
& \leq \Psi(J, K) \\
& \leq \Psi(q, J) .
\end{aligned}
$$

Therefore, $\Psi\left(q, \breve{g} p_{n}\right) \longrightarrow \Psi(q, J)$. In view of the fact that $J$ is approximately compact with respect to $K,\left\{\breve{g} p_{n}\right\}$ has a subsequence $\left\{\mathscr{g} p_{n_{k}}\right\}$ converging to some $z=g(p \in J$ for some $p \in J_{0}$. Thus,

$$
\Psi(z, q)=\lim _{k \longrightarrow \infty} \mathscr{D}\left(\breve{g} p_{n_{k}}, \check{T} p_{n_{k-1}}\right)=\Psi(J, K) .
$$

Therefore, $z$ is a member of $J_{0}$. Since $J_{0}$ is contained in $\breve{g}\left(J_{0}\right)$ and $z=\breve{g} p$ for some $p$ in $J_{0}, \breve{g} p_{n_{k}} \longrightarrow \breve{g} p$ and $\breve{g}$ is a one-to-one continuous mapping, so $p_{n_{k}} \longrightarrow p$. Since $T$ is continuous, it can be concluded that $T p_{n_{k}} \longrightarrow \check{T} p$. This implies that

$$
\mathscr{D}(\breve{g} p, \check{T} p)=\lim _{k \longrightarrow \infty} \mathscr{D}\left(\breve{g} p_{n_{k}}, \check{T} p_{n_{k-1}}\right)=\Psi(J, K) .
$$
$(\breve{g}, \check{T})$

That is, $p$ is a coincidence best proximity point of the pair

To prove the uniqueness of the coincidence best proximity point of the pair of mappings $(\breve{g}, \breve{T})$, suppose that there is another coincidence best proximity point $q \neq p$ of the pair $(\breve{g}, \check{T})$. We have

$$
\begin{aligned}
& \mathscr{D}(\breve{g} p, \check{T} p)=\Psi(J, K), \\
& \mathscr{D}(\breve{g} q, \check{T} q)=\Psi(J, K) .
\end{aligned}
$$

As the mapping $\check{T}$ is one-to-one on the set $J$ and $p \neq q$, one has $\mathscr{H}(\check{T} p, \check{T} q)>0$. Since the pair $(\breve{g}, \breve{T})$ is a $\beta$-generalized proximal contraction, one can write

$$
(0<) \mathscr{H}(\check{T} p, \check{T} q) \leq \beta \mathscr{H}(\check{T} p, \check{T} q)<\mathscr{H}(\check{T} p, \check{T} q) .
$$

It is a contradiction.

Corollary 1. Let $\check{T}: J \longrightarrow \mathscr{B}(K)$ and $\alpha_{*}: X \times X \longrightarrow$ $[1, \infty)$ be mappings, where $K$ is a closed subset and $J$ is approximately compact with respect to $K$ with $\check{T}\left(J_{0}\right) \subseteq K_{0}$. Suppose that $\check{T}$ is a continuous and $\beta_{\breve{T}}$-generalized proximal contraction such that

$$
\begin{aligned}
& \sup _{m \geq 1} \lim _{i \longrightarrow \infty} \alpha_{*}\left(p_{i}, p_{i+1}\right) \alpha_{*}\left(p_{i}, p_{m}\right)<\frac{1}{k}, \\
& \lim _{n \longrightarrow \infty} \alpha_{*}\left(p_{n}, \check{T} p_{n-1}\right)=1, \quad \text { where } k \in(0,1),
\end{aligned}
$$

then there exists a unique best proximity point of $\check{T}$.

Proof. If we take identity mapping $\breve{g}=I_{J}(\breve{g}$ is identity on $J)$, the remaining proof is same as in Theorem 3

Definition 16. Let $\check{T}: J \longrightarrow K$ and $\breve{g}: J \longrightarrow J$. A pair of mappings $(\breve{g}, \breve{T})$ is said to be a $\beta$-modified proximal contraction if there exists $\beta \in[0,1)$ such that

$$
\left.\begin{array}{l}
\Psi\left(\breve{g} u_{1}, \check{T} p_{1}\right)=\Psi(J, K) \\
\Psi\left(\breve{g} u_{2}, \check{T} p_{2}\right)=\Psi(J, K)
\end{array}\right\}, \quad \operatorname{implies} \Psi\left(\breve{T} u_{1}, \check{T} u_{2}\right) \leq \beta \Psi\left(\breve{T} p_{1}, \breve{T} p_{2}\right),
$$

for all $u_{1}, u_{2}, p_{1}$, and $p_{2}$ in $J$.

Definition 17. A mapping $\check{T}: J \longrightarrow K$ is said to be a $\beta_{\check{T}^{-}}$ modified proximal contraction if there exists $\beta \in[0,1)$ such that

$$
\left.\begin{array}{l}
\Psi\left(u_{1}, \check{T} p_{1}\right)=\Psi(J, K) \\
\Psi\left(u_{2}, \check{T} p_{2}\right)=\Psi(J, K)
\end{array}\right\}, \quad \text { implies } \Psi\left(\check{T} u_{1}, \check{T} u_{2}\right) \leq \beta \Psi\left(\check{T} p_{1}, \check{T} p_{2}\right),
$$

for all $u_{1}, u_{2}, p_{1}$, and $p_{2}$ in $J$.

Note that if we take $\breve{g}=I_{I}$ (the identity mapping on $J$ ), then every $\beta$-modified proximal contraction is a $\beta_{\breve{T}}$-modified proximal contraction.

Theorem 4. Let $\check{T}: J \longrightarrow K$ and $\breve{g}: J \longrightarrow J$ be two continuous and one-to-one mappings, where $K$ is a closed subset and $J$ is approximately compact with respect to $K$ with $\check{T}\left(J_{0}\right) \subseteq K_{0}$ and $J_{0} \subseteq \breve{g}\left(J_{0}\right)$. If the pair $(\breve{g}, \breve{T})$ is a $\beta$-modified proximal contraction and

$$
\begin{aligned}
& \sup _{m \geq 1} \lim _{i \longrightarrow \infty} \alpha\left(p_{i}, p_{i+1}\right) \alpha\left(p_{i}, p_{m}\right)<\frac{1}{k}, \\
& \lim _{n \longrightarrow \infty} \alpha\left(\breve{g} p_{n}, \check{T} p_{n-1}\right)=1, \quad \text { where } k \in(0,1),
\end{aligned}
$$

then there exists a unique coincidence best proximity point of the pair $(\breve{g}, \breve{T})$.

Proof. Let $p_{0}$ be an arbitrary element in $J_{0}$. Since $\check{T}\left(J_{0}\right)$ is contained in $K_{0}$ and $J_{0}$ is contained in $\breve{g}\left(J_{0}\right)$, there exists an element $p_{1}$ in $J_{0}$ such that

$$
\Psi\left(\breve{g} p_{1}, \check{T} p_{0}\right)=\Psi(J, K) .
$$

Since $\breve{T} p_{1}$ is an element of $\breve{T}\left(J_{0}\right)$ which is contained in $K_{0}$ and $J_{0}$ is contained in $\breve{g}\left(J_{0}\right)$, it follows that there exists an element $p_{2}$ in $J_{0}$ such that

$$
\Psi\left(\breve{g} p_{2}, \check{T} p_{1}\right)=\Psi(J, K) .
$$

By continuing this process, we can construct a sequence $\left\{p_{n}\right\}$ in $J_{0}$, satisfying the condition as follows:

$$
\Psi\left(\breve{g} p_{n}, \breve{T} p_{n-1}\right)=\Psi(J, K) .
$$

Having chosen $\left\{p_{n}\right\}$ in $J_{0}$, there exists an element $p_{n+1}$ in $J_{0}$, such that

$$
\Psi\left(\breve{g} p_{n+1}, \check{T} p_{n}\right)=\Psi(J, K)
$$


for every positive integer $n$. Since the pair $(\breve{g}, \breve{T})$ is a $\beta$ modified proximal contraction from equations (43) and (44), we obtain

$$
\Psi\left(\check{T} p_{n+1}, \check{T} p_{n}\right) \leq \beta \Psi\left(\check{T} p_{n}, \check{T} p_{n-1}\right) .
$$

Recursively, we have

$$
\Psi\left(\check{T} p_{n+1}, \check{T} p_{n}\right) \leq \beta^{n} \Psi\left(\check{T} p_{1}, \check{T} p_{0}\right) .
$$

Now, we have to prove that $\left\{\check{T} p_{n}\right\}$ is a Cauchy sequence. For all natural numbers $n, m \in \mathbb{N}$ with $n<m$, we have

$$
\begin{aligned}
& \Psi\left(\check{T} p_{n}, \check{T} p_{m}\right) \leq \alpha\left(\check{T} p_{n}, \check{T} p_{n+1}\right) \Psi\left(\check{T} p_{n}, \check{T} p_{n+1}\right)+\alpha\left(\check{T} p_{n+1}, \check{T} p_{m}\right) \Psi\left(\check{T} p_{n+1}, \check{T} p_{m}\right) \\
& \leq \alpha\left(\check{T} p_{n}, \check{T} p_{n+1}\right) \Psi\left(\check{T} p_{n}, \check{T} p_{n+1}\right)+\alpha\left(\check{T} p_{n+1}, \breve{T} p_{m}\right) \alpha\left(\check{T} p_{n+1}, \check{T} p_{n+2}\right) \Psi\left(\check{T} p_{n+1}, \check{T} p_{n+2}\right) \\
& +\alpha\left(\breve{T} p_{n+1}, \breve{T} p_{m}\right) \alpha\left(\check{T} p_{n+2}, \breve{T} p_{m}\right) \Psi\left(\breve{T} p_{n+2}, \check{T} p_{m}\right) \\
& \leq \alpha\left(\check{T} p_{n}, \check{T} p_{n+1}\right) \Psi\left(\check{T} p_{n}, \check{T} p_{n+1}\right)+\alpha\left(\check{T} p_{n+1}, \check{T} p_{m}\right) \alpha\left(\check{T} p_{n+1}, \check{T} p_{n+2}\right) \Psi\left(\check{T} p_{n+1}, \check{T} p_{n+2}\right) \\
& +\alpha\left(\check{T} p_{n+1}, \check{T} p_{m}\right) \alpha\left(\check{T} p_{n+2}, \check{T} p_{m}\right) \alpha\left(\check{T} p_{n+2}, \breve{T} p_{n+3}\right) \Psi\left(\check{T} p_{n+2}, \check{T} p_{n+3}\right)+\alpha\left(\check{T} p_{n+1}, \check{T} p_{m}\right) \\
& \alpha\left(\check{T} p_{n+2}, \check{T} p_{m}\right) \alpha\left(\check{T} p_{n+3}, \check{T} p_{m}\right) \Psi\left(\check{T} p_{n+3}, \check{T} p_{m}\right) \\
& \leq \alpha\left(\check{T} p_{n}, \check{T} p_{n+1}\right) \Psi\left(\check{T} p_{n}, \check{T} p_{n+1}\right)+\sum_{i=n+1}^{m-2}\left(\prod_{j=n+1}^{i} \alpha\left(\check{T} p_{j}, \check{T} p_{m}\right)\right) \alpha\left(\check{T} p_{i}, \check{T} p_{i+1}\right) \Psi\left(\check{T} p_{i}, \check{T} p_{i+1}\right) \\
& +\prod_{k=n+1}^{m-1} \alpha\left(\check{T} p_{k}, \check{T} p_{m}\right) \Psi\left(\check{T} p_{m-1}, \check{T} p_{m}\right) \\
& \leq \alpha\left(\check{T} p_{n}, \check{T} p_{n+1}\right) \beta^{n} \Psi\left(\check{T} p_{0}, \check{T} p_{1}\right)+\sum_{i=n+1}^{m-2}\left(\prod_{j=n+1}^{i} \alpha\left(\check{T} p_{j}, \check{T} p_{m}\right)\right) \alpha\left(\check{T} p_{i}, \check{T} p_{i+1}\right) \beta^{i} \Psi\left(\check{T} p_{0}, \check{T} p_{1}\right) \\
& +\prod_{k=n+1}^{m-1} \alpha\left(\check{T} p_{k}, \check{T} p_{m}\right) \beta^{m-1} \Psi\left(\check{T} p_{0}, \check{T} p_{1}\right) \\
& \leq \alpha\left(\check{T} p_{i}, \check{T} p_{i+1}\right) \beta^{n} \Psi\left(\check{T} p_{0}, \check{T} p_{1}\right)+\sum_{i=n+1}^{m-2}\left(\prod_{j=n+1}^{i} \alpha\left(\check{T} p_{j}, \check{T} p_{m}\right)\right) \alpha\left(\check{T} p_{i}, \check{T} p_{i+1}\right) \beta^{i} \Psi\left(\check{T} p_{0}, \check{T} p_{1}\right) \\
& +\prod_{k=n+1}^{m-1} \alpha\left(\check{T} p_{k}, \check{T} p_{m}\right) \alpha\left(\check{T} p_{m-1}, \check{T} p_{m}\right) \beta^{m-1} \Psi\left(\check{T} p_{0}, \check{T} p_{1}\right) \\
& =\alpha\left(\check{T} p_{n}, \check{T} p_{n+1}\right) \beta^{n} \Psi\left(\check{T} p_{0}, \check{T} p_{1}\right)+\sum_{i=n+1}^{m-1}\left(\prod_{j=n+1}^{i} \alpha\left(\check{T} p_{j}, \check{T} p_{m}\right)\right) \alpha\left(\check{T} p_{i}, \check{T} p_{i+1}\right) \beta^{i} \Psi\left(\check{T} p_{0}, \check{T} p_{1}\right) \\
& \leq \alpha\left(\check{T} p_{n}, \check{T} p_{n+1}\right) \beta^{n} \Psi\left(\check{T} p_{0}, \check{T} p_{1}\right)+\sum_{i=n+1}^{m-1}\left(\prod_{j=0}^{i} \alpha\left(\check{T} p_{j}, \check{T} p_{m}\right)\right) \alpha\left(\check{T} p_{i}, \check{T} p_{i+1}\right) \beta^{i} \Psi\left(\check{T} p_{0}, \check{T} p_{1}\right) . \\
& a_{i}=\prod_{j=0}^{i} \alpha\left(\check{T} p_{j}, \check{T} p_{m}\right) \alpha\left(\check{T} p_{i}, \check{T} p_{i+1}\right) \beta^{i}, \quad \text { where } \frac{a_{i+1}}{a_{i}}<\frac{1}{k}
\end{aligned}
$$

Assume that

$$
S_{l}=\sum_{i=0}^{l}\left(\prod_{j=0}^{i} \alpha\left(\check{T} p_{j}, \check{T} p_{m}\right)\right) \alpha\left(\check{T} p_{i}, \check{T} p_{i+1}\right) \beta^{i} .
$$

It follows that

$\Psi\left(\check{T} p_{n}, \check{T} p_{m}\right) \leq \Psi\left(\check{T} p_{0}, \check{T} p_{1}\right)\left[\beta^{n} \alpha\left(\check{T} p_{n}, \check{T} p_{n+1}\right)+\left(S_{m-1}-S_{n}\right)\right]$.

Using the ratio test, we have

By applying limit $m, n \longrightarrow \infty$ in inequality (49), we get

$$
\lim _{n \longrightarrow \infty} \Psi\left(\check{T} p_{n}, \check{T} p_{m}\right)=0,
$$

which shows that $\left\{\check{T} p_{n}\right\}$ is a Cauchy sequence; hence, it is convergent to some $q$ in $K$ (as the set $K$ is closed). Therefore, 


$$
\begin{aligned}
\Psi(q, J) & \leq \Psi\left(q, \breve{g} p_{n}\right) \leq \alpha\left(q, \check{T} p_{n-1}\right) \Psi\left(q, \check{T} p_{n-1}\right)+\alpha\left(\check{T} p_{n-1}, \breve{g} p_{n}\right) \Psi\left(\check{T} p_{n-1}, \breve{g} p_{n}\right) \\
& =\alpha\left(q, \check{T} p_{n-1}\right) \Psi\left(q, \check{T} p_{n-1}\right)+\alpha\left(\check{T} p_{n-1}, \breve{g} p_{n}\right) \Psi(J, K) .
\end{aligned}
$$

Taking limit $n \longrightarrow \infty$ on both sides of the above inequality, we have

$$
\begin{aligned}
\lim _{n \longrightarrow \infty} \Psi\left(q, \breve{g} p_{n}\right) & \leq \lim _{n \longrightarrow \infty}\left[\alpha\left(q, \check{T} p_{n-1}\right) \Psi\left(q, \check{T} p_{n-1}\right)+\alpha\left(\check{T} p_{n-1}, \breve{g} p_{n}\right) \Psi(J, K)\right] \\
& \leq \Psi(J, K) \\
& \leq \Psi(q, J) .
\end{aligned}
$$

Therefore, $\Psi\left(q, \breve{g} p_{n}\right) \longrightarrow \Psi(q, J)$. In view of the fact that $J$ is approximately compact with respect to $K,\left\{\breve{g} p_{n}\right\}$ has a subsequence $\left\{\tilde{g} p_{n_{k}}\right\}$ converging to some $z=g(p \in J$ for some $p \in J_{0}$. It follows that

$$
\Psi(z, q)=\lim _{k \longrightarrow \infty} \Psi\left(\breve{g} p_{n_{k}}, \check{T} p_{n_{k-1}}\right)=\Psi(J, K) .
$$

Therefore, $z$ is an element of $J_{0}$. Since $J_{0}$ is contained in $\breve{g}\left(J_{0}\right)$, we have $z=\breve{g} p$ for some $p$ in $J_{0}$. As $\breve{g} p_{n_{k}} \longrightarrow \breve{g} p$ and $g$ is a one-to-one continuous mapping, $p_{n_{k}} \longrightarrow p$. Since $\check{T}$ is continuous, it can be concluded that $\breve{T} p_{n_{k}} \longrightarrow \breve{T} p$. Hence,

$$
\Psi(\breve{g} p, \breve{T} p)=\lim _{k \longrightarrow \infty} \Psi\left(\breve{g} p_{n_{k}}, \check{T} p_{n_{k-1}}\right)=\Psi(J, K) .
$$

To prove the uniqueness, suppose that $q$ is another coincidence best proximity point of the pair $(\breve{g}, \breve{T})$ such that $p \neq q$. Then,

$$
\begin{aligned}
& \Psi(\breve{g} p, \check{T} p)=\Psi(J, K), \\
& \Psi(\breve{g} q, \check{T} q)=\Psi(J, K) .
\end{aligned}
$$

Since the pair $(\breve{g}, \check{T})$ is a $\beta$-modified proximal contraction, we have

$$
0<\Psi(\check{T} p, \check{T} q) \leq \beta \Psi(\check{T} p, \check{T} q)<\Psi(\check{T} p, \check{T} q),
$$

which is a contradiction (as $\check{T}$ is one-to-one mapping on $J$ ). Hence, the pair $(\breve{g}, \check{T})$ has a unique coincidence best proximity point.

Corollary 2. Let $\check{T}: J \longrightarrow K$ be a given continuous mapping, where $K$ is a closed subset and $J$ is approximately compact with respect to $K$ with $\breve{T}\left(J_{0}\right) \subseteq K_{0}$. If $\breve{T}$ is a $\beta_{\breve{T}}$-modified proximal contraction and suppose that

$$
\begin{aligned}
& \sup _{m \geq 1} \lim _{i \longrightarrow \infty} \alpha\left(p_{i}, p_{i+1}\right) \alpha\left(p_{i}, p_{m}\right)<\frac{1}{k}, \\
& \lim _{n \longrightarrow \infty} \alpha\left(p_{n}, \check{T} p_{n-1}\right)=1, \quad \text { where } k \in(0,1),
\end{aligned}
$$

then there exists a unique best proximity point of $\check{T}$.

Proof. If we take $\breve{g}=I_{J}$ (the identity mapping over the set $J)$, the remaining proof is same as Theorem 4 .

Example 1. Let $X=\{0,1,2,3,4,5\}$. Consider the function $\Psi$ given as $\Psi(p, p)=0$ and $\Psi(p, q)=\Psi(q, p)$, where

\begin{tabular}{|c|c|c|c|c|c|c|}
\hline$\Psi$ & 0 & 1 & 2 & 3 & 4 & 5 \\
\hline 0 & 0 & $1 / 14$ & $1 / 13$ & $1 / 15$ & $1 / 12$ & $1 / 11$ \\
\hline 1 & $1 / 14$ & 0 & $2 / 3$ & $3 / 4$ & $1 / 15$ & $4 / 5$ \\
\hline 2 & $1 / 13$ & $2 / 3$ & 0 & $1 / 9$ & $1 / 8$ & $1 / 15$ \\
\hline 3 & $1 / 15$ & $3 / 4$ & $1 / 9$ & 0 & $7 / 8$ & $8 / 9$ \\
\hline 4 & $1 / 12$ & $1 / 15$ & $1 / 8$ & $7 / 8$ & 0 & $1 / 4$ \\
\hline 5 & $1 / 11$ & $4 / 5$ & $1 / 15$ & $8 / 9$ & $1 / 4$ & 0 \\
\hline
\end{tabular}

Take $\alpha: X \times X \longrightarrow[1, \infty)$ to be symmetric which is defined as $\alpha(p, q)=19 p+21 q$. It is easy to see that $(X, \Psi)$ is a controlled metric type space. Take $J=\{0,1,2\}$ and $K=\{3,4,5\}$. Obviously, $\Psi(J, K)=(1 / 15), \quad J_{0}=J$, and $K_{0}=K$. Now, consider $\check{T}: J \longrightarrow K$ as follows:

$$
\check{T} p= \begin{cases}3, & \text { if } p=\{0,1\}, \\ 5, & \text { if } p=2 .\end{cases}
$$

Clearly, $\breve{T}\left(J_{0}\right) \subseteq K_{0}$. Define $\breve{g}$ : $J \longrightarrow J$ by

$$
\breve{g} p= \begin{cases}0, & \text { if } p=0, \\ 1, & \text { if } p=2, \\ 2, & \text { if } p=1 .\end{cases}
$$

We get $J_{0} \subseteq \breve{g}\left(J_{0}\right)$. Now, we have to show that the pair $(\breve{g}, \check{T})$ satisfies

$$
\begin{aligned}
& \Psi(\breve{g} 0, \check{T} 1)=\Psi(0,3)=\Psi(J, K), \\
& \Psi(\breve{g} 1, \check{T} 2)=\Psi(2,5)=\Psi(J, K),
\end{aligned}
$$

where $u_{1}=0, u_{2}=1, p_{1}=1$, and $p_{2}=2$. Since the pair $(\breve{g}, \check{T})$ is a $\beta$-modified proximal contraction:

$$
\Psi(\check{T} 0, \check{T} 1) \leq \beta \Psi(\check{T} 1, \check{T} 2),
$$

for every $\beta \in[0,1)$, the pair $(\breve{g}, \breve{T})$ is a $\beta$-modified proximal contraction. Hence, 0 is the unique coincidence best proximity point of $\check{T}$ and $\breve{g}$.

Definition 18. Let $\check{T}: J \longrightarrow K$ and $\breve{g}: J \longrightarrow J$. A pair of mappings $(\breve{g}, \breve{T})$ is said to be a $\beta$ - proximal contraction if there exists $\beta \in[0,1)$ such that

$$
\left.\begin{array}{l}
\Psi\left(\breve{g} u_{1}, \breve{T} p_{1}\right)=\Psi(J, K) \\
\Psi\left(\breve{g} u_{2}, \check{T} p_{2}\right)=\Psi(J, K)
\end{array}\right\}, \quad \text { implies } \Psi\left(\breve{g} u_{1}, \breve{g} u_{2}\right) \leq \beta \Psi\left(p_{1}, p_{2}\right),
$$

for all $u_{1}, u_{2}, p_{1}$, and $p_{2}$ in $J$.

Definition 19. A mapping $\check{T}: J \longrightarrow K$ is said to be a $\beta_{\breve{T}^{-}}$ proximal contraction if there exists $\beta \in[0,1)$ such that

$$
\left.\begin{array}{l}
\Psi\left(u_{1}, \check{T} p_{1}\right)=\Psi(J, K) \\
\Psi\left(u_{2}, \check{T} p_{2}\right)=\Psi(J, K)
\end{array}\right\}, \quad \operatorname{implies} \Psi\left(u_{1}, u_{2}\right) \leq \beta \Psi\left(p_{1}, p_{2}\right),
$$

for all $u_{1}, u_{2}, p_{1}$, and $p_{2}$ in $J$. 
Note that, if we take $\breve{g}=I_{J}$, then every $\beta$-proximal contraction is a $\beta_{\check{T}}$-proximal contraction.

Theorem 5. Let $\check{T}: J \longrightarrow K$ and $\breve{g}: J \longrightarrow J$ be continuous mappings, where $K$ is a closed subset and $J$ is approximately compact with respect to $K$ with $\breve{T}\left(J_{0}\right) \subseteq K_{0}$ and $J_{0} \subseteq \breve{g}\left(J_{0}\right)$. Suppose that $\breve{g}$ is an expansive mapping and the pair $(\breve{g}, \breve{T})$ is a $\beta$-proximal contraction such that

$$
\begin{aligned}
& \sup _{m \geq 1} \lim _{i \longrightarrow \infty} \alpha\left(p_{i}, p_{i+1}\right) \alpha\left(p_{i}, p_{m}\right)<\frac{1}{k}, \\
& \lim _{n \longrightarrow \infty} \alpha\left(\breve{g} p_{n}, \breve{T} p_{n-1}\right)=1, \quad \text { where } k \in(0,1),
\end{aligned}
$$

then there exists a unique coincidence best proximity point of the pair $(\breve{g}, \breve{T})$.

Proof. Let $p_{0}$ be an arbitrary element in $J_{0}$. Since $\breve{T}\left(J_{0}\right)$ is contained in $K_{0}$ and $J_{0}$ is contained in $\breve{g}\left(J_{0}\right)$, there exists an element $p_{1}$ in $J_{0}$ such that

$$
\Psi\left(\breve{g} p_{1}, \check{T} p_{0}\right)=\Psi(J, K) .
$$

Again, since $\breve{T} p_{1}$ is an element of $\breve{T}\left(J_{0}\right)$ which is contained in $K_{0}$ and $J_{0}$ is contained in $\breve{g}\left(J_{0}\right)$, it follows that there is an element $p_{2}$ in $J_{0}$, such that

$$
\Psi\left(\breve{g} p_{2}, \check{T} p_{1}\right)=\Psi(J, K) .
$$
that

This process can be continued by selecting $p_{n}$ in $J_{0}$ so

$$
\Psi\left(\breve{g} p_{n+1}, \check{T} p_{n}\right)=\Psi(J, K) .
$$

Since the pair $(\breve{g}, \breve{T})$ is a $\beta$-proximal contraction, we have

$$
\Psi\left(\breve{g} p_{n+1}, \breve{g} p_{n}\right) \leq \beta \Psi\left(p_{n}, p_{n-1}\right) .
$$

As $\breve{g}$ is an expansive mapping, one writes

$$
\Psi\left(p_{n+1}, p_{n}\right) \leq \Psi\left(\breve{g} p_{n+1}, \breve{g} p_{n}\right) \leq \beta^{n} \Psi\left(p_{1}, p_{0}\right),
$$

so we have

$$
\Psi\left(p_{n+1}, p_{n}\right) \leq \beta^{n} \Psi\left(p_{1}, p_{0}\right) .
$$

We claim that $\left\{p_{n}\right\}$ is a Cauchy sequence. For all natural numbers $n, m \in \mathbb{N}$ with $n<m$, we have

$$
\begin{aligned}
\Psi\left(p_{n}, p_{m}\right) \leq & \alpha\left(p_{n}, p_{n+1}\right) \Psi\left(p_{n}, p_{n+1}\right)+\alpha\left(p_{n+1}, p_{m}\right) \Psi\left(p_{n+1}, p_{m}\right) \\
\leq & \alpha\left(p_{n}, p_{n+1}\right) \Psi\left(p_{n}, p_{n+1}\right)+\alpha\left(p_{n+1}, p_{m}\right) \alpha\left(p_{n+1}, p_{n+2}\right) \Psi\left(p_{n+1}, p_{n+2}\right) \\
& +\alpha\left(p_{n+1}, p_{m}\right) \alpha\left(p_{n+2}, p_{m}\right) \Psi\left(p_{n+2}, p_{m}\right) \\
\leq & \alpha\left(p_{n}, p_{n+1}\right) \Psi\left(p_{n}, p_{n+1}\right)+\alpha\left(p_{n+1}, p_{m}\right) \alpha\left(p_{n+1}, p_{n+2}\right) \Psi\left(p_{n+1}, p_{n+2}\right) \\
& +\alpha\left(p_{n+1}, p_{m}\right) \alpha\left(p_{n+2}, p_{m}\right) \alpha\left(p_{n+2}, p_{n+3}\right) \Psi\left(p_{n+2}, p_{n+3}\right)+\alpha\left(p_{n+1}, p_{m}\right) \\
\alpha\left(p_{n+2}, p_{m}\right) \alpha\left(p_{n+3}, p_{m}\right) \Psi\left(p_{n+3}, p_{m}\right) & \\
\leq & \alpha\left(p_{n}, p_{n+1}\right) \Psi\left(p_{n}, p_{n+1}\right)+\sum_{i=n+1}^{m-2}\left(\prod_{j=n+1}^{i} \alpha\left(p_{j}, p_{m}\right)\right) \alpha\left(p_{i}, p_{i+1}\right) \Psi\left(p_{i}, p_{i+1}\right) \\
& +\prod_{k=n+1}^{m-1} \alpha\left(p_{k}, p_{m}\right) \Psi\left(p_{m-1}, p_{m}\right) \\
\leq & \alpha\left(\check{T}_{p_{n}}, \check{T}_{p_{n+1}}\right) \beta^{n} \Psi\left(p_{0}, p_{1}\right)+\sum_{i=n+1}^{m-2}\left(\prod_{j=n+1}^{i} \alpha\left(p_{j}, p_{m}\right)\right) \alpha\left(p_{i}, p_{i+1}\right) \beta^{i} \Psi\left(p_{0}, p_{1}\right) \\
& +\prod_{k=n+1}^{m-1} \alpha\left(p_{k}, p_{m}\right) \beta^{m-1} \Psi\left(p_{0}, p_{1}\right) \\
\leq & \alpha\left(p_{n}, p_{n+1}\right) \beta^{n} \Psi\left(p_{0}, p_{1}\right)+\sum_{i=n+1}^{m-2}\left(\prod_{j=n+1}^{i} \alpha\left(p_{j}, p_{m}\right)\right) \alpha\left(p_{i}, p_{i+1}\right) \beta^{i} \Psi\left(p_{0}, p_{1}\right) \\
& +\prod_{k=n+1}^{m-1} \alpha\left(p_{k}, p_{m}\right) \alpha\left(p_{m-1}, p_{m}\right) \beta^{m-1} \Psi\left(p_{0}, p_{1}\right) \\
= & \alpha\left(p_{n}, p_{n+1}\right) \beta^{n} \Psi\left(p_{0}, p_{1}\right)+\sum_{i=n+1}^{m-1}\left(\prod_{j=n+1}^{i} \alpha\left(p_{j}, p_{m}\right)\right) \alpha\left(p_{i}, p_{i+1}\right) \beta^{i} \Psi\left(p_{0}, p_{1}\right) \\
\leq & \alpha\left(p_{n}, p_{n+1}\right) \beta^{n} \Psi\left(p_{0}, p_{1}\right)+\sum_{i=n+1}^{m-1}\left(\prod_{j=0}^{i} \alpha\left(p_{j}, p_{m}\right)\right) \alpha\left(p_{i}, p_{i+1}\right) \beta^{i} \Psi\left(p_{0}, p_{1}\right) . \\
&
\end{aligned}
$$


Assume that

$$
S_{l}=\sum_{i=0}^{l}\left(\prod_{j=0}^{i} \alpha\left(p_{j}, p_{m}\right)\right) \alpha\left(p_{i}, p_{i+1}\right) \beta^{i}
$$

Then, we obtain

$$
\Psi\left(p_{n}, p_{m}\right) \leq \Psi\left(p_{0}, p_{1}\right)\left[\beta^{n} \alpha\left(p_{n}, p_{n+1}\right)+\left(S_{m-1}-S_{n}\right)\right] .
$$

Using the ratio test, we have

$a_{i}=\prod_{j=0}^{i} \alpha\left(p_{j}, p_{m}\right) \alpha\left(p_{i}, p_{i+1}\right) \beta^{i}, \quad$ where $\frac{a_{i+1}}{a_{i}}<\frac{1}{k}$.

By taking limit as $n, m \longrightarrow \infty$, (74) becomes

$$
\lim _{n \rightarrow \infty} \Psi\left(p_{n}, p_{m}\right)=0 \text {. }
$$

Therefore, $\left\{p_{n}\right\}$ is a Cauchy sequence in the complete controlled metric type space $(X, \Psi)$; hence, it is convergent to some $p$ in $J$ (as set $J$ is closed). Since $\breve{g}$ and $\check{T}$ are continuous, we have

$$
\Psi(\breve{g} p, \breve{T} p)=\lim _{n \longrightarrow \infty} \Psi\left(\breve{g} p_{n+1}, \check{T} p_{n}\right)=\Psi(J, K) .
$$

Hence, $p$ is the unique coincidence best proximity point of the pair $(\breve{g}, \breve{T})$. To prove the uniqueness, suppose that $q$ is another coincidence best proximity point of the pair $(\breve{g}, \breve{T})$ such that $p \neq q$. Then,

$$
\begin{aligned}
& \Psi(\breve{g} p, \check{T} p)=\Psi(J, K), \\
& \Psi(\breve{g} q, \check{T} q)=\Psi(J, K) .
\end{aligned}
$$

Since the pair $(\breve{g}, \check{T})$ is a $\beta$-modified proximal contraction, we have

$$
\Psi(p, q) \leq \Psi(\breve{g} p, \breve{g} q) \leq \beta \Psi(p, q)<\Psi(p, q),
$$

which is a contradiction. Hence, the pair $(\breve{g}, \check{T})$ has a unique coincidence best proximity point.
Corollary 3. Let $\check{T}: J \longrightarrow K$ be a continuous mapping, where $K$ is closed subset and $J$ is approximately compact with respect to $K$ with $\check{T}\left(J_{0}\right) \subseteq K_{0}$. If $\check{T}$ is a $\beta_{\check{T}}$-proximal contraction and suppose that

$$
\begin{aligned}
& \sup _{m \geq 1} \lim _{i \longrightarrow \infty} \alpha\left(p_{i}, p_{i+1}\right) \alpha\left(p_{i}, p_{m}\right)<\frac{1}{k}, \\
& \lim _{n \longrightarrow \infty} \alpha\left(p_{n}, \check{T} p_{n-1}\right)=1, \quad \text { where } k \in(0,1),
\end{aligned}
$$

then there exists a best proximity point of $\check{T}$.

Proof. If we take identity mapping $\breve{g}=I_{J}$, the remaining proof is same as Theorem 5 .

\section{Coincidence Best Proximity Points for Geraghty Type Proximal Contractive Mappings}

First, we need to define a generalized Geraghty type proximal contractive mapping.

From now and onward, $F$ is a class of all nondecreasing functions $\lambda:[0, \infty) \longrightarrow[0,1)$ such that for any bounded sequence $\left\{t_{n}\right\}$ of positive real numbers, $\lambda\left\{t_{n}\right\} \longrightarrow 1$ implies $t_{n} \longrightarrow 0$.

Definition 20. Let $(X, \Psi)$ be a controlled metric type space having a pair of nonempty subsets $(J, K)$ such that $J_{0}$ is nonempty. Then, a pair $(J, K)$ has the $P$-property if and only if

$$
\left.\begin{array}{l}
\Psi\left(p_{1}, q_{1}\right)=\Psi(J, K) \\
\Psi\left(p_{2}, q_{2}\right)=\Psi(J, K)
\end{array}\right\} \quad \text { implies } \Psi\left(p_{1}, p_{2}\right)=\Psi\left(q_{1}, q_{2}\right) .
$$

Definition 21. Let $\breve{T}: J \longrightarrow \mathscr{B}(K), \breve{g}: J \longrightarrow J$ are mappings. A pair $(\breve{g}, \check{T})$ is said to be a $\lambda-\mu$-proximal Geraghty contraction if $\mu: J \times J \longrightarrow[0, \infty)$ is such that

$$
\left.\begin{array}{l}
\mu(p, q) \geq 1 \\
\mathscr{D}(\check{g} u, \check{T} p)=\Psi(J, K) \\
\mathscr{D}(\check{g} v, \check{T} q)=\Psi(J, K)
\end{array}\right\} \quad \text { implies that } \mu(p, q) \mathscr{H}(\check{T} p, \check{T} q) \leq \lambda(M(u, v, p, q)) M(u, v, p, q) \text {, }
$$

where

$$
\begin{aligned}
M(u, v, p, q)= & \max \left\{\Psi(\breve{g} p, \breve{g} q), \frac{\mathscr{D}(\breve{g} p, \check{T} p)-\alpha_{*}(\breve{g} q, \breve{T} p) \Psi(J, K)}{\alpha_{*}(\breve{g} p, \breve{g} q)},\right. \\
& \left.\mathscr{D}^{*}(\breve{g} u, \check{T} p), \frac{\mathscr{D}(\breve{g} u, \check{T} q)-\alpha_{*}(\breve{g} v, \check{T} q) \Psi(J, K)}{\alpha_{*}(\breve{g} u, \breve{g} v)}\right\},
\end{aligned}
$$

for all $u, v, p, q \in J$, where $\lambda \in F$. 
Definition 22. A mapping $\check{T}: J \longrightarrow \mathscr{B}(K)$ is said to be a $(\lambda-\mu)_{\breve{T}}$-proximal Geraghty contraction if $\mu: J \times J \longrightarrow[0, \infty)$ is such that

$$
\left.\begin{array}{l}
\mu(p, q) \geq 1 \\
\mathscr{D}(u, \check{T} p)=\Psi(J, K) \\
\mathscr{D}(v, \check{T} q)=\Psi(J, K)
\end{array}\right\}, \quad \text { implies that } \mu(p, q) \mathscr{H}(\check{T} p, \check{T} q) \leq \lambda(M(u, v, p, q)) M(u, v, p, q) \text {, }
$$

where

$$
\begin{array}{r}
M(u, v, p, q)=\max \left\{\Psi(p, q), \frac{\Psi(p, \check{T} p)-\alpha_{*}(q, \check{T} p) \Psi(J, K)}{\alpha_{*}(p, q)},\right. \\
\left.\Psi^{*}(u, \check{T} p), \frac{\Psi(u, \check{T} q)-\alpha_{*}(v, \check{T} q) \Psi(J, K)}{\alpha_{*}(u, v)}\right\},
\end{array}
$$

$\sup _{m \geq 1} \lim _{i \longrightarrow \infty} \alpha_{*}\left(p_{i}, p_{i+1}\right) \alpha_{*}\left(p_{i}, p_{m}\right)<\frac{1}{k}, \quad$ where $k \in(0,1)$,

then the pair $(\breve{g}, \check{T})$ has a unique coincidence best proximity point $p^{*} \in J$.

for all $u, v, p, q \in J$, where $\lambda \in F$.

If we take $\breve{g}=I_{J}$ (the identity mapping over $J$ ), then every $\lambda-\mu$-proximal Geraghty contraction will reduce to a $\lambda-\mu$-generalized proximal Geraghty contraction.

Theorem 6. Let $\check{T}: J \longrightarrow \mathscr{B}(K), \quad \breve{g}: J \longrightarrow J$, and $\mu: J \times J \longrightarrow[0,+\infty)$ be mappings, where $J$ is a closed subset and the pair $(J, K)$ satisfies the P-property with $\check{T}\left(J_{0}\right) \subseteq K_{0}$ and $J_{0} \subseteq \mathscr{g}\left(J_{0}\right)$. If a pair of continuous mappings $(\breve{g}, \check{T})$ is

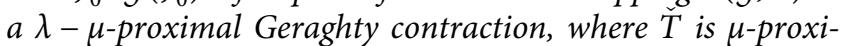
mal admissible, then there exist elements $p_{0}, p_{1} \in J_{0}$ such that $\mathscr{D}\left(\breve{g} p_{1}, \check{T} p_{0}\right)=\Psi(J, K)$ and $\mu\left(p_{0}, p_{1}\right) \geq 1$. If $\left\{p_{n}\right\}$ is a sequence in $J$ such that $\mu\left(p_{n}, p_{n+1}\right) \geq 1$ and suppose that

Proof. From the given condition, there exist $p_{0}, p_{1} \in J_{0}$ such that $\mathscr{D}\left(\breve{g} p_{1}, \check{T} p_{0}\right)=\Psi(J, K)$ and $\mu\left(p_{0}, p_{1}\right) \geq 1$. As $\check{T}\left(J_{0}\right) \subseteq K_{0}$, there exists $p_{2} \in J_{0}$ such that $\mathscr{D}\left(\check{g} p_{2}, \breve{T} p_{1}\right)=\Psi(J, K)$. As $\check{T}$ is $\mu$-proximal admissible, $\mu\left(p_{0}, p_{1}\right) \geq 1$,

$$
\begin{aligned}
& \mathscr{D}\left(\breve{g} p_{1}, \check{T} p_{0}\right)=\Psi(J, K), \\
& \mathscr{D}\left(\breve{g} p_{2}, \check{T} p_{1}\right)=\Psi(J, K),
\end{aligned}
$$

using the $P$-property $\Psi\left(\breve{g} p_{1}, \breve{g} p_{2}\right)=\mathscr{H}\left(\check{T} p_{0}, \check{T} p_{1}\right)$. Since the pair $(\breve{g}, \breve{T})$ is a $\lambda-\mu$-proximal Geraghty contraction with $\mu\left(p_{1}, p_{2}\right) \geq 1$, we have

$$
\Psi\left(\breve{g} p_{1}, \breve{g} p_{2}\right) \leq \lambda\left(M\left(p_{0}, p_{1}, p_{1}, p_{2}\right)\right) M\left(p_{0}, p_{1}, p_{1}, p_{2}\right),
$$

where

$$
\begin{aligned}
M\left(p_{0}, p_{1}, p_{1}, p_{2}\right) \leq & \max \left\{\Psi\left(\breve{g} p_{0}, \breve{g} p_{1}\right), \frac{\mathscr{D}\left(\breve{g} p_{0}, \breve{T} p_{0}\right)-\alpha_{*}\left(\breve{g} p_{1}, \breve{T} p_{0}\right) \Psi(J, K)}{\alpha_{*}\left(\breve{g} p_{0}, \breve{g} p_{1}\right)},\right. \\
& \left.\mathscr{D}^{*}\left(\breve{g} p_{1}, \breve{T} p_{0}\right), \frac{\mathscr{D}\left(\breve{g} p_{1}, \breve{T} p_{1}\right)-\alpha_{*}\left(\breve{g} p_{2}, \breve{T} p_{1}\right) \Psi(J, K)}{\alpha_{*}\left(\breve{g} p_{1}, \breve{g} p_{2}\right)}\right\} \\
\leq & \max \left\{\Psi\left(\breve{g} p_{0}, \breve{g} p_{1}\right), \frac{\alpha_{*}\left(\breve{g} p_{0}, \breve{g} p_{1}\right) \Psi\left(\breve{g} p_{0}, \breve{g} p_{1}\right)+\alpha_{*}\left(\breve{g} p_{1}, \check{T} p_{0}\right) \mathscr{D}\left(\breve{g} p_{1}, \breve{T} p_{0}\right)}{\alpha_{*}\left(\breve{g} p_{0}, \breve{g} p_{1}\right)}\right. \\
& -\frac{\alpha_{*}\left(\breve{g} p_{1}, \breve{T} p_{0}\right) \Psi(J, K)}{\alpha_{*}\left(\breve{g} p_{0}, \breve{g} p_{1}\right)}, \mathscr{D}\left(\breve{g} p_{1}, \breve{T} p_{0}\right)-\Psi(J, K), \frac{\alpha_{*}\left(\breve{g} p_{1}, \breve{g} p_{2}\right) \Psi\left(\breve{g} p_{1}, \breve{g} p_{2}\right)}{\alpha_{*}\left(\breve{g} p_{1}, \breve{g} p_{2}\right)} \\
& +\frac{\alpha_{*}\left(\breve{g} p_{2}, \check{T} p_{1}\right) \mathscr{D}\left(\breve{g} p_{2}, \check{T} p_{1}\right)-\alpha_{*}\left(\breve{g} p_{2}, \check{T} p_{1}\right) \Psi(J, K)}{\alpha_{*}\left(\breve{g} p_{1}, \breve{g} p_{2}\right)} \\
\leq & \max \left\{\Psi\left(\breve{g} p_{0}, \breve{g} p_{1}\right), \Psi\left(\breve{g} p_{0}, \breve{g} p_{1}\right), 0, \Psi\left(\breve{g} p_{1}, \breve{g} p_{2}\right)\right\},
\end{aligned}
$$


and we have

$$
M\left(p_{0}, p_{1}, p_{1}, p_{2}\right) \leq \max \left\{\Psi\left(\breve{g} p_{0}, \breve{g} p_{1}\right), \Psi\left(\breve{g} p_{1}, \breve{g} p_{2}\right)\right\} .
$$

If

$$
\max \left\{\Psi\left(\breve{g} p_{0}, \breve{g} p_{1}\right), \Psi\left(\breve{g} p_{1}, \breve{g} p_{2}\right)\right\}=\Psi\left(\breve{g} p_{1}, \breve{g} p_{2}\right),
$$

then inequality (88) becomes

$$
\Psi\left(\breve{g} p_{1}, \breve{g} p_{2}\right) \leq \lambda\left(\Psi\left(\breve{g} p_{1}, \breve{g} p_{2}\right)\right) \Psi\left(\breve{g} p_{1}, \breve{g} p_{2}\right),
$$

which is a contradiction. So, we can conclude that

$$
\Psi\left(\breve{g} p_{1}, \breve{g} p_{2}\right) \leq \lambda\left(\Psi\left(\breve{g} p_{0}, \breve{g} p_{1}\right)\right) \Psi\left(\breve{g} p_{0}, \breve{g} p_{1}\right) .
$$

Further, by the fact that $\check{T}\left(J_{0}\right) \subseteq K_{0}$, there exists $p_{3} \in J_{0}$ such that $\mathscr{D}\left(\breve{g} p_{3}, \check{T} p_{2}\right)=\Psi(J, K)$. As $\check{T}$ is $\mu$-proximal admissible mapping, where $\mu\left(p_{2}, p_{3}\right) \geq 1$,

$$
\begin{aligned}
& \mathscr{D}\left(\breve{g} p_{2}, \breve{T} p_{1}\right)=\Psi(J, K), \\
& \mathscr{D}\left(\breve{g} p_{3}, \check{T} p_{2}\right)=\Psi(J, K),
\end{aligned}
$$

using the $P$-Property, we have $\Psi\left(\breve{g} p_{2}, \breve{g} p_{3}\right)=\mathscr{H}\left(\check{T} p_{1}, \check{T} p_{2}\right)$. Since the pair $(\breve{g}, \breve{T})$ is a $\lambda-\mu$-proximal Geraghty mapping with $\mu\left(p_{2}, p_{3}\right) \geq 1$, one writes

$$
\Psi\left(\breve{g} p_{2}, \breve{g} p_{3}\right) \leq \lambda\left(M\left(p_{2}, p_{3}, p_{1}, p_{2}\right)\right) M\left(p_{2}, p_{3}, p_{1}, p_{2}\right),
$$

where

$$
\begin{aligned}
M\left(p_{2}, p_{3}, p_{1}, p_{2}\right) \leq & \max \left\{\Psi\left(\breve{g} p_{1}, \breve{g} p_{2}\right), \frac{\mathscr{D}\left(\breve{g} p_{1}, \breve{T} p_{1}\right)-\alpha_{*}\left(\breve{g} p_{2}, \breve{T} p_{1}\right) \Psi(J, K)}{\alpha_{*}\left(\breve{g} p_{1}, \breve{g} p_{2}\right)},\right. \\
& \left.\mathscr{D}^{*}\left(\breve{g} p_{2}, \check{T} p_{1}\right), \frac{\mathscr{D}\left(\breve{g} p_{2}, \check{T} p_{2}\right)-\alpha_{*}\left(\breve{g} p_{3}, \breve{T} p_{2}\right) \Psi(J, K)}{\alpha_{*}\left(\breve{g} p_{2}, \breve{g} p_{3}\right)}\right\} \\
\leq & \max \left\{\Psi\left(\breve{g} p_{1}, \breve{g} p_{2}\right), \frac{\alpha_{*}\left(\breve{g} p_{1}, \breve{g} p_{2}\right) \Psi\left(\breve{g} p_{1}, \breve{g} p_{2}\right)+\alpha_{*}\left(\breve{g} p_{2}, \check{T} p_{1}\right) \mathscr{D}\left(\breve{g} p_{2}, \check{T} p_{1}\right)}{\alpha_{*}\left(\breve{g} p_{1}, \breve{g} p_{2}\right)}\right. \\
& -\frac{\alpha_{*}\left(\breve{g} p_{2}, \check{T} p_{1}\right) \Psi(J, K)}{\alpha_{*}\left(\breve{g} p_{1}, \breve{g} p_{2}\right)}, \mathscr{D}\left(\breve{g} p_{2}, \check{T} p_{1}\right)-\Psi(J, K), \frac{\alpha_{*}\left(\breve{g} p_{2}, \breve{g} p_{3}\right) \Psi\left(\breve{g} p_{2}, \breve{g} p_{3}\right)}{\alpha_{*}\left(\breve{g} p_{2}, \breve{g} p_{3}\right)} \\
& \left.+\frac{\alpha_{*}\left(\breve{g} p_{3}, \breve{T} p_{2}\right) \mathscr{D}\left(\breve{g} p_{3}, \check{T} p_{2}\right)-\alpha_{*}\left(\breve{g} p_{3}, \check{T} p_{2}\right) \Psi(J, K)}{\alpha_{*}\left(\breve{g} p_{2}, \breve{g} p_{3}\right)}\right\} \\
\leq & \max \left\{\Psi\left(\breve{g} p_{1}, \breve{g} p_{2}\right), \Psi\left(\breve{g} p_{1}, \breve{g} p_{2}\right), 0, \Psi\left(\breve{g} p_{2}, \breve{g} p_{3}\right)\right\},
\end{aligned}
$$

and we have

$$
M\left(p_{2}, p_{3}, p_{1}, p_{2}\right) \leq \max \left\{\Psi\left(\breve{g} p_{1}, \breve{g} p_{2}\right), \Psi\left(\breve{g} p_{2}, \breve{g} p_{3}\right)\right\} .
$$

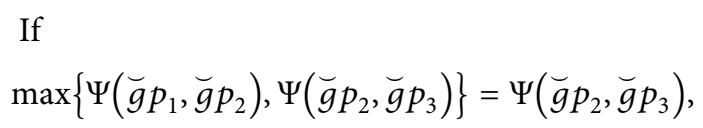

then inequality (95) becomes

$$
\Psi\left(\breve{g} p_{2}, \breve{g} p_{3}\right) \leq \lambda\left(\Psi\left(\breve{g} p_{2}, \breve{g} p_{3}\right)\right) \Psi\left(\breve{g} p_{2}, \breve{g} p_{3}\right),
$$

which is a contradiction. Thus,

$$
\Psi\left(\breve{g} p_{2}, \breve{g} p_{3}\right) \leq \lambda\left(\Psi\left(\breve{g} p_{1}, \breve{g} p_{2}\right)\right) \Psi\left(\breve{g} p_{1}, \breve{g} p_{2}\right) .
$$

Similarly, we can construct a sequence $\left\{p_{n}\right\} \subseteq J_{0}$, where $\mu\left(p_{n}, p_{n+1}\right) \geq 1$ for all $n \in \mathbb{N} \cup\{0\}$,

$$
\begin{aligned}
& \mathscr{D}\left(\breve{g} p_{n}, \breve{T} p_{n-1}\right)=\Psi(J, K), \\
& \mathscr{D}\left(\breve{g} p_{n+1}, \check{T} p_{n}\right)=\Psi(J, K),
\end{aligned}
$$

using the $P$-property $\Psi\left(\breve{g} p_{n}, \breve{g} p_{n+1}\right)=\mathscr{H}\left(\check{T} p_{n}, \check{T} p_{n-1}\right)$. Since the pair $(\breve{g}, \check{T})$ is a $\lambda-\mu$-proximal Geraghty contraction with $\mu\left(p_{n}, p_{n+1}\right) \geq 1$, we get

$$
\Psi\left(\breve{g} p_{n}, \breve{g} p_{n+1}\right) \leq \lambda\left(M\left(p_{n}, p_{n+1}, p_{n-1}, p_{n}\right)\right) M\left(p_{n}, p_{n+1}, p_{n-1}, p_{n}\right),
$$

where 


$$
\begin{aligned}
& M\left(p_{n}, p_{n+1}, p_{n-1}, p_{n}\right) \leq \max \left\{\Psi\left(\breve{g} p_{n-1}, \breve{g} p_{n}\right), \frac{\mathscr{D}\left(\breve{g} p_{n-1}, \breve{T} p_{n-1}\right)-\alpha_{*}\left(\breve{g} p_{n}, \breve{T} p_{n-1}\right) \Psi(J, K)}{\alpha_{*}\left(\breve{g} p_{n-1}, \breve{g} p_{n}\right)}\right. \\
& \left.\mathscr{D}^{*}\left(\breve{g} p_{n}, \check{T} p_{n-1}\right), \frac{\mathscr{D}\left(\breve{g} p_{n}, \check{T} p_{n}\right)-\alpha_{*}\left(\breve{g} p_{n+1}, \check{T} p_{n}\right) \Psi(J, K)}{\alpha_{*}\left(\breve{g} p_{n}, \breve{g} p_{n+1}\right)}\right\} \\
& \leq \max \left\{\Psi\left(\breve{g} p_{n-1}, \breve{g} p_{n}\right), \frac{\alpha_{*}\left(\breve{g} p_{n-1}, \breve{g} p_{n}\right) \Psi\left(\breve{g} p_{n-1}, \breve{g} p_{n}\right)+\alpha_{*}\left(\breve{g} p_{n}, \check{T} p_{n-1}\right) \mathscr{D}\left(\breve{g} p_{n}, \check{T} p_{n-1}\right)}{\alpha_{*}\left(\breve{g} p_{n-1}, \breve{g} p_{n}\right)}\right. \\
& -\frac{\alpha_{*}\left(\breve{g} p_{n}, \check{T} p_{n-1}\right) \Psi(J, K)}{\alpha_{*}\left(\breve{g} p_{n-1}, \breve{g} p_{n}\right)} \mathscr{D}\left(\breve{g} p_{n}, \check{T} p_{n-1}\right)-\Psi(J, K) \\
& \left.\frac{\alpha_{*}\left(\breve{g} p_{n}, \breve{g} p_{n+1}\right) \Psi\left(\breve{g} p_{n}, \breve{g} p_{n+1}\right)+\alpha_{*}\left(\left(g p_{n+1}, \breve{T} p_{n}\right) \mathscr{D}\left(\breve{g} p_{n+1}, \check{T} p_{n}\right)\right.}{\alpha_{*}\left(\breve{g} p_{n}, \breve{g} p_{n+1}\right)}-\frac{\alpha_{*}\left(\breve{g} p_{n+1}, \check{T} p_{n}\right) \Psi(J, K)}{\alpha_{*}\left(\breve{g} p_{n}, \breve{g} p_{n+1}\right)}\right\} \\
& \leq \max \left\{\Psi\left(\breve{g} p_{n-1}, \breve{g} p_{n}\right), \Psi\left(\breve{g} p_{n-1}, \breve{g} p_{n}\right), 0, \Psi\left(\breve{g} p_{n}, \breve{g} p_{n+1}\right)\right\} . \\
& M\left(p_{n}, p_{n+1}, p_{n-1}, p_{n}\right) \leq \max \left\{\Psi\left(\breve{g} p_{n-1}, \breve{g} p_{n}\right), \Psi\left(\breve{g} p_{n}, \breve{g} p_{n+1}\right)\right\} . \\
& \lambda\left(\Psi\left(\breve{g} p_{n}, \breve{g} p_{n+1}\right)\right) \leq \lambda\left(\Psi\left(\breve{g} p_{n-1}, \breve{g} p_{n}\right)\right), \\
& \lambda\left(\Psi\left(\breve{g} p_{n-1}, \breve{g} p_{n}\right)\right) \leq \lambda\left(\Psi\left(\breve{g} p_{n-2}, \breve{g} p_{n-1}\right)\right) .
\end{aligned}
$$

If

$$
\max \left\{\Psi\left(\breve{g} p_{n-1}, \breve{g} p_{n}\right), \Psi\left(\breve{g} p_{n}, \breve{g} p_{n+1}\right)\right\}=\Psi\left(\breve{g} p_{n}, \breve{g} p_{n+1}\right)
$$

then inequality (102) becomes

$$
\Psi\left(\breve{g} p_{n}, \breve{g} p_{n+1}\right) \leq \lambda\left(\Psi\left(\breve{g} p_{n}, \breve{g} p_{n+1}\right)\right) \Psi\left(\breve{g} p_{n}, \breve{g} p_{n+1}\right),
$$

which is a contradiction. So, we conclude that

$$
\Psi\left(\breve{g} p_{n}, \breve{g} p_{n+1}\right) \leq \lambda\left(\Psi\left(\breve{g} p_{n-1}, \breve{g} p_{n}\right)\right) \Psi\left(\breve{g} p_{n-1}, \breve{g} p_{n}\right) .
$$

Further,

$$
\Psi\left(\breve{g} p_{n}, \breve{g} p_{n+1}\right) \leq \lambda\left(\Psi\left(\breve{g} p_{n-1}, \breve{g} p_{n}\right)\right) \Psi\left(\breve{g} p_{n-1}, \breve{g} p_{n}\right) \leq \Psi\left(\breve{g} p_{n-1}, \breve{g} p_{n}\right),
$$

Continuing on the same lines, we can write $\lambda\left(\Psi\left(\breve{g} p_{n-1}, \breve{g} p_{n}\right)\right) \leq \lambda\left(\Psi\left(\breve{g} p_{n-2}, \breve{g} p_{n-1}\right)\right) \leq \cdots \leq \lambda\left(\Psi\left(\breve{g} p_{0}, \breve{g} p_{1}\right)\right)$.

Using inequality (107),

$$
\Psi\left(\breve{g} p_{n-1}, \breve{g} p_{n}\right) \leq \lambda\left(\Psi\left(\breve{g} p_{n-2}, \breve{g} p_{n-1}\right)\right) \Psi\left(\breve{g} p_{n-2}, \breve{g} p_{n-1}\right) .
$$

From inequalities (107) and (111), we have

$$
\begin{aligned}
\Psi\left(\breve{g} p_{n}, \breve{g} p_{n+1}\right) \leq & \lambda\left(\Psi\left(\breve{g} p_{n-1}, \breve{g} p_{n}\right)\right) \Psi\left(\breve{g} p_{n-1}, \breve{g} p_{n}\right) \\
\leq & \lambda\left(\Psi\left(\breve{g} p_{n-1}, \breve{g} p_{n}\right)\right) \lambda\left(\Psi\left(\breve{g} p_{n-2}, \breve{g} p_{n-1}\right)\right) \\
& \cdot \Psi\left(\breve{g} p_{n-2}, \breve{g} p_{n-1}\right) .
\end{aligned}
$$

Following on similar lines, we have

which shows that $\left\{\Psi\left(\breve{g} p_{n}, \breve{g} p_{n+1}\right)\right\}$ is a decreasing sequence.

Since $\lambda \in F$, from (108), we have

$$
\begin{aligned}
\Psi\left(\breve{g} p_{n}, \breve{g} p_{n+1}\right) & \leq \lambda\left(\Psi\left(\breve{g} p_{n-1}, \breve{g} p_{n}\right)\right) \lambda\left(\Psi\left(\breve{g} p_{n-2}, \breve{g} p_{n-1}\right)\right), \ldots, \lambda\left(\Psi\left(\breve{g} p_{0}, \breve{g} p_{1}\right)\right) \Psi\left(\breve{g} p_{0}, \breve{g} p_{1}\right) \\
& =\lambda^{n}\left(\Psi\left(\breve{g} p_{0}, \breve{g} p_{1}\right)\right) \Psi\left(\breve{g} p_{0}, \breve{g} p_{1}\right) .
\end{aligned}
$$

We deduce

$$
\Psi\left(\breve{g} p_{n}, \breve{g} p_{n+1}\right) \leq \lambda^{n}\left(\Psi\left(\breve{g} p_{0}, \breve{g} p_{1}\right)\right) \Psi\left(\breve{g} p_{0}, \breve{g} p_{1}\right)
$$

From (108), suppose that $\Psi\left(\breve{g} p_{n-1}, \breve{g} p_{n}\right)>0$, so we can conclude
$\frac{\Psi\left(\breve{g} p_{n}, \breve{g} p_{n+1}\right)}{\Psi\left(\breve{g} p_{n-1}, \breve{g} p_{n}\right)} \leq \lambda\left(\Psi\left(\breve{g} p_{n-1}, \breve{g} p_{n}\right)\right) \leq 1, \quad$ for all $n \geq 1$.

Let $l=\lim _{n \longrightarrow+\infty} \Psi\left(\breve{g} p_{n-1}, \breve{g} p_{n}\right)$. Using equation (108) and letting $n \longrightarrow+\infty$, we obtain that 


$$
\bar{l}=1 \leq \lim _{n \longrightarrow+\infty} \lambda\left(\Psi\left(\breve{g} p_{n-1}, \breve{g} p_{n}\right)\right) \leq 1 .
$$

Thus, $\lim _{n \longrightarrow+\infty} \Psi\left(\breve{g} p_{n-1}, \breve{g} p_{n}\right)=1$. Using the definition of $\lambda$, we conclude that

$$
\lim _{n \longrightarrow+\infty} \Psi\left(\breve{g} p_{n-1}, \breve{g} p_{n}\right)=0 .
$$

Now, we have to show that $\left\{\breve{g} p_{n}\right\}$ is a Cauchy sequence. For all natural numbers $n, m \in \mathbb{N}$ with $n<m$, we have

$$
\begin{aligned}
& \Psi\left(\breve{g} p_{n}, \breve{g} p_{m}\right) \leq \alpha_{*}\left(\breve{g} p_{n}, \breve{g} p_{n+1}\right) \Psi\left(\breve{g} p_{n}, \breve{g} p_{n+1}\right)+\alpha_{*}\left(\breve{g} p_{n+1}, \breve{g} p_{m}\right) \Psi\left(\breve{g} p_{n+1}, \breve{g} p_{m}\right) \\
& \leq \alpha_{*}\left(\breve{g} p_{n}, \breve{g} p_{n+1}\right) \Psi\left(\breve{g} p_{n}, \breve{g} p_{n+1}\right)+\alpha_{*}\left(\breve{g} p_{n+1}, \breve{g} p_{m}\right) \alpha_{*}\left(\breve{g} p_{n+1}, \breve{g} p_{n+2}\right) \Psi\left(\breve{g} p_{n+1}, \breve{g} p_{n+2}\right) \\
& +\alpha_{*}\left(\breve{g} p_{n+1}, \breve{g} p_{m}\right) \alpha_{*}\left(\breve{g} p_{n+2}, \breve{g} p_{m}\right) \Psi\left(\breve{g} p_{n+2}, \breve{g} p_{m}\right) \\
& \leq \alpha_{*}\left(\breve{g} p_{n}, \breve{g} p_{n+1}\right) \Psi\left(\breve{g} p_{n}, \breve{g} p_{n+1}\right)+\alpha_{*}\left(\breve{g} p_{n+1}, \breve{g} p_{m}\right) \alpha_{*}\left(\breve{g} p_{n+1}, \breve{g} p_{n+2}\right) \Psi\left(\breve{g} p_{n+1}, \breve{g} p_{n+2}\right) \\
& +\alpha_{*}\left(\breve{g} p_{n+1}, \breve{g} p_{m}\right) \alpha_{*}\left(\left(g p_{n+2}, \breve{g} p_{m}\right) \alpha_{*}\left(\breve{g} p_{n+2}, \breve{g} p_{n+3}\right) \Psi\left(\breve{g} p_{n+2}, \breve{g} p_{n+3}\right)+\alpha_{*}\left(\breve{g} p_{n+1}, \breve{g} p_{m}\right)\right. \\
& \alpha_{*}\left(\breve{g} p_{n+2}, \breve{g} p_{m}\right) \alpha_{*}\left(\breve{g} p_{n+3}, \breve{g} p_{m}\right) \Psi\left(\breve{g} p_{n+3}, \breve{g} p_{m}\right) \\
& \leq \alpha_{*}\left(\breve{g} p_{n}, \breve{g} p_{n+1}\right) \Psi\left(\breve{g} p_{n}, \breve{g} p_{n+1}\right) \\
& +\sum_{i=n+1}^{m-2}\left(\prod_{j=n+1}^{i} \alpha_{*}\left(\breve{g} p_{j}, \breve{g} p_{m}\right)\right) \alpha_{*}\left(\breve{g} p_{i}, \breve{g} p_{i+1}\right) \Psi\left(\breve{g} p_{i}, \breve{g} p_{i+1}\right)+\prod_{k=n+1}^{m-1} \alpha_{*}\left(\breve{g} p_{k}, \breve{g} p_{m}\right) \Psi\left(\breve{g} p_{m-1}, \breve{g} p_{m}\right) \\
& \leq \alpha_{*}\left(\breve{g} p_{n}, \breve{g} p_{n+1}\right) \lambda^{n}\left(\Psi\left(\breve{g} p_{0}, \breve{g} p_{1}\right)\right) \Psi\left(\breve{g} p_{0}, \breve{g} p_{1}\right)+\sum_{i=n+1}^{m-2}\left(\prod_{j=n+1}^{i} \alpha_{*}\left(\breve{g} p_{j}, \breve{g} p_{m}\right)\right) \alpha_{*}\left(\breve{g} p_{i}, \breve{g} p_{i+1}\right) \\
& \lambda^{i}\left(\Psi\left(\breve{g} p_{0}, \breve{g} p_{1}\right)\right) \Psi\left(\breve{g} p_{0}, \breve{g} p_{1}\right)+\prod_{k=n+1}^{m-1} \alpha_{*}\left(\breve{g} p_{k}, \breve{g} p_{m}\right) \lambda^{m-1}\left(\Psi\left(\breve{g} p_{0}, \breve{g} p_{1}\right)\right) \Psi\left(\breve{g} p_{0}, \breve{g} p_{1}\right) \\
& \leq \alpha_{*}\left(\breve{g} p_{n}, \breve{g} p_{n+1}\right) \lambda^{n}\left(\Psi\left(\breve{g} p_{0}, \breve{g} p_{1}\right)\right) \Psi\left(\breve{g} p_{0}, \breve{g} p_{1}\right) \\
& +\sum_{i=n+1}^{m-2}\left(\prod_{j=n+1}^{i} \alpha_{*}\left(\breve{g} p_{j}, \breve{g} p_{m}\right)\right) \alpha_{*}\left(\breve{g} p_{i}, \breve{g} p_{i+1}\right) \lambda\left(\Psi\left(\breve{g} p_{0}, \breve{g} p_{1}\right)\right) \Psi\left(\breve{g} p_{0}, \breve{g} p_{1}\right) \\
& +\prod_{k=n+1}^{m-1} \alpha_{*}\left(\breve{g} p_{k}, \breve{g} p_{m}\right) \alpha_{*}\left(\breve{g} p_{m-1}, \breve{g} p_{m}\right) \\
& \lambda^{m-1}\left(\Psi\left(\breve{g} p_{0}, \breve{g} p_{1}\right)\right) \Psi\left(\breve{g} p_{0}, \breve{g} p_{1}\right)=\alpha_{*}\left(\breve{g} p_{n}, \breve{g} p_{n+1}\right) \lambda^{n}\left(\Psi\left(\breve{g} p_{0}, \breve{g} p_{1}\right)\right) \Psi\left(\breve{g} p_{0}, \breve{g} p_{1}\right) \\
& +\sum_{i=n+1}^{m-1}\left(\prod_{j=n+1}^{i} \alpha_{*}\left(\breve{g} p_{j}, \breve{g} p_{m}\right)\right) \alpha_{*}\left(\breve{g} p_{i}, \breve{g} p_{i+1}\right) \lambda^{i}\left(\Psi\left(\breve{g} p_{0}, \breve{g} p_{1}\right)\right) \Psi\left(\breve{g} p_{0}, \breve{g} p_{1}\right) \\
& \leq \alpha_{*}\left(\breve{g} p_{n}, \breve{g} p_{n+1}\right) \lambda^{n}\left(\Psi\left(\breve{g} p_{0}, \breve{g} p_{1}\right)\right) \Psi\left(\breve{g} p_{0}, \breve{g} p_{1}\right) \\
& +\sum_{i=n+1}^{m-1}\left(\prod_{j=0}^{i} \alpha_{*}\left(\breve{g} p_{j}, \breve{g} p_{m}\right)\right) \alpha_{*}\left(\breve{g} p_{i}, \breve{g} p_{i+1}\right) \\
& \lambda^{i}\left(\Psi\left(\breve{g} p_{0}, \breve{g} p_{1}\right)\right) \Psi\left(\breve{g} p_{0}, \breve{g} p_{1}\right) \text {. }
\end{aligned}
$$

Assume that

$S_{l}=\sum_{i=0}^{l}\left(\prod_{j=0}^{i} \alpha_{*}\left(\breve{g} p_{j}, \breve{g} p_{m}\right)\right) \alpha_{*}\left(\breve{g} p_{i}, \breve{g} p_{i+1}\right) \lambda^{i}\left(\Psi\left(\breve{g} p_{0}, \breve{g} p_{1}\right)\right)$.
Then, we obtain

$$
\begin{aligned}
\Psi\left(\breve{g} p_{n}, \breve{g} p_{m}\right) \leq & \Psi\left(\breve{g} p_{0}, \breve{g} p_{1}\right)\left[\lambda^{n}\left(\Psi\left(\breve{g} p_{0}, \breve{g} p_{1}\right)\right) \alpha_{*}\left(\breve{g} p_{n}, \breve{g} p_{n+1}\right)\right. \\
& \left.+\left(S_{m-1}-S_{n}\right)\right] .
\end{aligned}
$$


Using the ratio test, we have

$$
\begin{aligned}
& a_{i}=\prod_{j=0}^{i} \alpha_{*}\left(\breve{g} p_{j}, \breve{g} p_{m}\right) \alpha_{*}\left(\breve{g} p_{i}, \breve{g} p_{i+1}\right) \lambda^{i}\left(\Psi\left(\breve{g} p_{0}, \breve{g} p_{1}\right)\right), \\
& \quad \text { where } \frac{a_{i+1}}{a_{i}}<\frac{1}{k}
\end{aligned}
$$

Taking limit as $n, m \longrightarrow \infty$, inequality (120) becomes

$$
\lim _{n, m \longrightarrow \infty} \Psi\left(\breve{g} p_{n}, \breve{g} p_{m}\right)=0
$$

This implies that $\left\{\breve{g} p_{n}\right\}$ is a Cauchy sequence in the complete controlled metric type space $(X, \Psi)$; hence, it is convergent and suppose that it converges to some $p^{*}$ in $J_{0} \subseteq J$ (as set $J$ is closed), which assures that the sequence $\left\{p_{n}\right\} \subseteq J_{0}$ since $p_{n} \longrightarrow p^{*}$. As $(\breve{g}, \check{T})$ is a pair of continuous mappings, one writes

$$
\mathscr{D}\left(\breve{g} p^{*}, \check{T} p^{*}\right)=\Psi(J, K)
$$

Therefore, $p^{*}$ is a coincidence best proximity point of the pair $(\breve{g}, \breve{T})$.

For uniqueness, suppose that there are two distinct coincidence best proximity points of $(\breve{g}, \breve{T})$ such that $p^{*} \neq q^{*}$. Thus, $s=\Psi\left(p^{*}, q^{*}\right)>0$. Since $\Psi\left(\breve{g} p^{*}, \check{T} p^{*}\right)=$ $\Psi\left(\breve{g} q^{*}, \check{T} q^{*}\right)=\Psi(J, K)$, using the $P$-property, we conclude that $s=\mathscr{H}\left(\check{T} p^{*}, \check{T} q^{*}\right)$. Since the pair $(\breve{g}, \check{T})$ is a $\lambda-\mu$ proximal Geraghty contraction, we obtain $s \leq \lambda(s) s$. Thus, $\lambda(s) \geq 1$. Since $\lambda(s) \geq 1$, we conclude that $\lambda(s)=1$ and therefore $s=0$, which is contradiction.

Example 2. Let $X=\{0,1,2,3,4,5\}$ be endowed with the function $\Psi$ given as $\Psi(p, q)=\Psi(q, p)$ and $\Psi(p, p)=0$, where

\begin{tabular}{|c|c|c|c|c|c|c|}
\hline$\Psi$ & 0 & 1 & 2 & 3 & 4 & 5 \\
\hline 0 & 0 & $1 / 2$ & $1 / 3$ & $1 / 10$ & $1 / 5$ & $1 / 6$ \\
\hline 1 & $1 / 2$ & 0 & $1 / 4$ & $2 / 3$ & $1 / 10$ & $3 / 4$ \\
\hline 2 & $1 / 3$ & $1 / 4$ & 0 & $6 / 7$ & $7 / 8$ & $1 / 10$ \\
\hline 3 & $1 / 10$ & $2 / 3$ & $6 / 7$ & 0 & $1 / 2$ & $1 / 3$ \\
\hline 4 & $1 / 5$ & $1 / 10$ & $7 / 8$ & $1 / 2$ & 0 & $1 / 4$ \\
\hline 5 & $1 / 6$ & $3 / 4$ & $1 / 10$ & $1 / 3$ & $1 / 4$ & 0 \\
\hline
\end{tabular}

Take $\alpha: X \times X \longrightarrow[1, \infty)$ to be symmetric and defined as $\alpha(p, q)=16 p+18 q$. It is easy to see that $(X, \Psi)$ is controlled type metric space. Suppose $J=\{0,1,2\}$ and $K=\{3,4,5\}$. After a simple calculation, $\Psi(J, K)=(1 / 10)$, the $P$-property is satisfied, $J_{0}=J$, and $K_{0}=K$. Consider

$$
\begin{aligned}
& \check{T} p= \begin{cases}3, & \text { if } p=2, \\
\{3,4\}, & \text { if } p=\{0,1\},\end{cases} \\
& \breve{g} p= \begin{cases}0, & \text { if } p=0, \\
1, & \text { if } p=2, \\
2, & \text { if } p=1 .\end{cases}
\end{aligned}
$$

Clearly, $\check{T}\left(J_{0}\right) \subseteq K_{0}$ and $J_{0} \subseteq \breve{g}\left(J_{0}\right)$. Now, we have to show that the pair $(\breve{g}, \breve{T})$ is a $\lambda-\mu$-proximal Geraghty contraction:

$$
\mu(p, q) \mathscr{H}(\check{T} p, \check{T} q) \leq \lambda(M(u, v, p, q)) M(u, v, p, q),
$$

for all $u, v, p, q \in J$ and for the function $\mu: J \times J \longrightarrow[0,+\infty)$ is defined by

$$
\mu(p, q)=\Psi(p, q)+1
$$

Hence,

$$
\begin{aligned}
& \mathscr{D}(\breve{g} 0, \check{T} 2)=\mathscr{D}(1,3)=\Psi(J, K), \\
& \mathscr{D}(\breve{g} 2, \check{T} 1)=\mathscr{D}(1,\{3,4\})=\Psi(J, K) .
\end{aligned}
$$

After simple calculations, $\mathscr{H}(\check{T} p, \check{T} q)=\mathscr{H}(3,\{3,4\})=0$, $\mu(p, q)=\Psi(3,\{3,4\})+1=1$, and

$$
\begin{aligned}
M(0,2,2,1)= & \max \left\{\Psi(\breve{g} 2, \breve{g} 1), \frac{\mathscr{D}(\breve{g} 2, \check{T} 2)-\alpha_{*}(\breve{g} 1, \check{T} 2)(1 / 10)}{\alpha_{*}(\breve{g} 2, \breve{g} 1)}\right. \\
& \left.\mathscr{D}^{*}(\breve{g} 0, \check{T} 2), \frac{\mathscr{D}(\breve{g} 0, \check{T} 1)-\alpha_{*}(\breve{g} 2, \check{T} 1)(1 / 10)}{\alpha_{*}(\breve{g} 0, \breve{g} 2)}\right\} \\
= & \max \left\{\Psi(1,2), \frac{\mathscr{D}(1,3)-\alpha_{*}(2,3)(1 / 10)}{\alpha_{*}(1,2)}\right. \\
& \left.\mathscr{D}^{*}(0,3), \frac{\mathscr{D}(0,\{3,4\})-\alpha_{*}(1,\{3,4\})(1 / 10)}{\alpha_{*}(0,1)}\right\} \\
= & \max \left\{\frac{1}{4}, \frac{-238}{1560}, 0, \frac{-69}{180}\right\}=\frac{1}{4} .
\end{aligned}
$$


Now, we have to show that the pair $(\breve{g}, \check{T})$ is a $\lambda-\mu$ proximal Geraghty contraction:

$$
\begin{aligned}
(1)(0) & \leq \lambda(M(u, v, p, q))\left(\frac{1}{4}\right) \\
& \leq \lambda(M(u, v, p, q))\left(\frac{1}{4}\right),
\end{aligned}
$$

and for every $\lambda:[0, \infty) \longrightarrow[0,1]$, the pair $(\breve{g}, \check{T})$ is a $\lambda-\mu$ proximal Geraghty contraction. Hence, 0 is the unique coincidence point of the pair of mappings $(\breve{g}, \breve{T})$.

Corollary 4. Let $\check{T}: J \longrightarrow \mathscr{B}(K)$ be a continuous mapping, where $J$ is a closed subset and the pair $(J, K)$ satisfies the P-property with $\check{T}\left(J_{0}\right) \subseteq K_{0}$. If $\check{T}$ is a $(\lambda-\mu)_{\check{T}}$-proximal Geraghty contraction, where $\check{T}$ is $\mu$-proximal admissible, then there exist elements $p_{0}, p_{1} \in J_{0}$ such that $\mathscr{D}\left(p_{1}, \check{T} p_{0}\right)=\Psi(J, K)$ and $\mu\left(p_{0}, p_{1}\right) \geq 1$. If $\left\{p_{n}\right\}$ is a sequence in $J$ such that $\mu\left(p_{n}, p_{n+1}\right) \geq 1$ and suppose that

$$
\sup _{m \geq 1} \lim _{i \longrightarrow \infty} \alpha_{*}\left(p_{i}, p_{i+1}\right) \alpha_{*}\left(p_{i}, p_{m}\right)<\frac{1}{k}, \quad \text { where } k \in(0,1) \text {, }
$$

then $\check{T}$ has a unique best proximity point $p^{*} \in J$.

Proof. If we take $\breve{g}=I_{J}$ (an identity mapping over $J$ ), the remaining proof is same as Theorem 6 .

Definition 23. Let $\check{T}: J \longrightarrow K, \breve{g}: J \longrightarrow J$, and $\mu: J \times J \longrightarrow$ $[0,+\infty)$ be mappings. A pair of mappings $(\breve{g}, \breve{T})$ is said to be a $\lambda$ - $\mu$-modified proximal Geraghty contraction if

$$
\left.\begin{array}{l}
\mu(p, q) \geq 1 \\
\Psi(\breve{g} u, \check{T} p)=\Psi(J, K) \\
\Psi(\breve{g} v, \check{T} q)=\Psi(J, K)
\end{array}\right\}, \quad \text { implies } \mu(p, q) \Psi(\check{T} p, \check{T} q) \leq \lambda(M(u, v, p, q)) M(u, v, p, q),
$$

where

$$
\begin{array}{r}
M(u, v, p, q)=\max \left\{\Psi(\breve{g} p, \breve{g} q), \frac{\Psi(\breve{g} p, \check{T} p)-\alpha(\breve{g} q, \check{T} p) \Psi(J, K)}{\alpha(\breve{g} p, \breve{g} q)},\right. \\
\left.\Psi^{*}(\breve{g} u, \check{T} p), \frac{\Psi(\breve{g} u, \check{T} q)-\alpha(\breve{g} v, \check{T} q) \Psi(J, K)}{\alpha(\breve{g} u, \breve{g} v)}\right\},
\end{array}
$$

for all $u, v, p, q \in J$, where $\lambda \in F$.

$$
\left.\begin{array}{l}
\mu(p, q) \geq 1 \\
\Psi(u, \check{T} p)=\Psi(J, K) \\
\Psi(v, \check{T} q)=\Psi(J, K)
\end{array}\right\}, \quad \text { implies } \mu(p, q) \Psi(\check{T} p, \check{T} q) \leq \lambda(M(u, v, p, q)) M(u, v, p, q)
$$

Definition 24. Let $\check{T}: J \longrightarrow K$ and $\mu: J \times J \longrightarrow[0,+\infty)$ be mappings. A mapping $\check{T}$ is said to be a $(\lambda-\mu)_{\breve{T}}$-modified proximal Geraghty contraction if

where

$$
\begin{array}{r}
M(u, v, p, q)=\max \left\{\Psi(p, q), \frac{\Psi(p, \check{T} p)-\alpha(q, \check{T} p) \Psi(J, K)}{\alpha(p, q)},\right. \\
\left.\Psi^{*}(u, \check{T} p), \frac{\Psi(u, \check{T} q)-\alpha(v, \check{T} q) \Psi(J, K)}{\alpha(u, v)}\right\},
\end{array}
$$


for all $u, v, p, q \in J$, where $\lambda \in F$.

Note that, if we take $\breve{g}=I_{J}$ (an identity mapping over $J$ ), then every $\lambda-\mu$-modified proximal Geraghty contraction will reduce to a $(\lambda-\mu)_{\check{T}}$-modified proximal Geraghty contraction.

Theorem 7. Let $\check{T}: J \longrightarrow K$ and $\breve{g}: J \longrightarrow J$ be continuous mappings, where $J$ is closed subset and the pair $(J, K)$ satisfies the P-property with $\breve{T}\left(J_{0}\right) \subseteq K_{0}$ and $J_{0} \subseteq \breve{g}\left(J_{0}\right)$. If the pair of

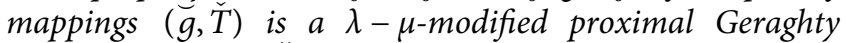
contraction, where $\breve{T}$ is a $\mu$-proximal admissible, then there exist elements $p_{0}, p_{1} \in J_{0}$ such that $\Psi\left(\breve{g} p_{1}, \breve{T} p_{0}\right)=\Psi(J, K)$ and $\mu\left(p_{0}, p_{1}\right) \geq 1$. If $\left\{p_{n}\right\}$ is a sequence in $J$ such that $\mu\left(p_{n}, p_{n+1}\right) \geq 1$ and suppose that

$$
\sup _{m \geq 1} \lim _{i \longrightarrow \infty} \alpha\left(p_{i}, p_{i+1}\right) \alpha\left(p_{i}, p_{m}\right)<\frac{1}{k}, \quad \text { where } k \in(0,1),
$$

then the pair $(\breve{g}, \check{T})$ has a unique coincidence best proximity point $p^{*} \in J$.

Proof. It is a simple consequence of Theorem 6.

Corollary 5. Let $\check{T}: J \longrightarrow K$ be a continuous mapping, where $J$ is closed subset and the pair $(J, K)$ satisfies the P-property with $\check{T}\left(J_{0}\right) \subseteq K_{0}$. If $\check{T}$ is a $(\lambda-\mu)_{\breve{T}}$-modified proximal Geraghty contraction, where $\check{T}$ is a $\mu$-proximal admissible, then there exist elements $p_{0}, p_{1} \in J_{0}$ such that $\Psi\left(\breve{g} p_{1}, \breve{T} p_{0}\right)=\Psi(J, K)$ and $\mu\left(p_{0}, p_{1}\right) \geq 1$. If $\left\{p_{n}\right\}$ is a sequence in $J$ such that $\mu\left(p_{n}, p_{n+1}\right) \geq 1$ and suppose that

$$
\sup _{m \geq 1} \lim _{i \longrightarrow \infty} \alpha\left(p_{i}, p_{i+1}\right) \alpha\left(p_{i}, p_{m}\right)<\frac{1}{k}, \quad \text { where } k \in(0,1),
$$

then the pair $(\breve{g}, \breve{T})$ has a unique best proximity point $p^{*} \in J$.

Proof. If we take $\breve{g}=I_{J}$, the remaining proof is same as Theorem 7.

\section{Conclusion}

In our paper, we ensured the existence of some best proximity point results via the multivalued concept in controlled metric spaces. To our knowledge, we are the first who worked on best proximity points for the class of multivalued mappings in this setting. We open the door for new perspectives when dealing with new generalized multivalued (proximal) contractions.

\section{Data Availability}

The data used to support the findings of this study are available from the corresponding author upon request.

\section{Conflicts of Interest}

The authors declare that they have no conflicts of interest concerning the publication of this article.

\section{Authors' Contributions}

All authors contributed equally and significantly for writing this article. All authors read and approved the final manuscript.

\section{References}

[1] K. Fan, "Extensions of two fixed point theorems of F. E. Browder," Mathematische Zeitschrift, vol. 112, no. 3, pp. 234-240, 1969.

[2] J. Anuradha and P. Veeramani, "Proximal pointwise contraction," Topology and Its Applications, vol. 156, no. 18, pp. 2942-2948, 2009.

[3] N. Saleem, I. Iqbal, B. Iqbal, and S. Radenovi'c, "Coincidence and fixed points of multivalued $F$-contractions in generalized metric space with application," Journal of Fixed Point Theory and Applications, vol. 22, 2020.

[4] S. Sadiq Basha, "Extensions of banach's contraction principle," Numerical Functional Analysis and Optimization, vol. 31, no. 5, pp. 569-576, 2010.

[5] W. A. Kirk, S. Reich, and P. Veeramani, "Proximinal retracts and best proximity pair theorems," Numerical Functional Analysis and Optimization, vol. 24, no. 7-8, pp. 851-862, 2003.

[6] V. Sankar Raj, "A best proximity point theorem for weakly contractive non-self-mappings," Nonlinear Analysis: Theory, Methods \& Applications, vol. 74, no. 14, pp. 4804-4808, 2011.

[7] P. S. Srinivasan, "Analysis-Best proximity pair theorems," Acta Scientiarum Mathematicarum, vol. 67, no. 1-2, pp. 421-430, 2001.

[8] T. Suzuki, M. Kikkawa, and C. Vetro, "The existence of best proximity points in metric spaces with the property UC, Nonlinear Analysis Theory," Methods \& Applications, vol. 71, no. 7-8, pp. 2918-2926, 2009.

[9] C. Vetro and T. Suzuki, "Three existence theorems for weak contractions of Matkowski type," International Journal of Mathematics and Statistics, pp. 110-220, 2010.

[10] M. Simkhah, D. Turkoglu, S. Sedghi, and N. Shobe, "Suzuki type fixed point results in $p$-metric spaces," Communications in Optimization Theory, Article ID 13, 2019.

[11] E. Naraghirad, "Bregman best proximity points for Bregman asymptotic cyclic contraction mappings in Banach spaces," Journal of Nonlinear and Variational Analysis, vol. 3, pp. 27-44, 2019.

[12] V. Parvaneh, M. R. Haddadi, and H. Aydi, "On best proximity point results for some type of mappings," Journal of Function Spaces, vol. 2020, Article ID 6298138, 6 pages, 2020.

[13] H. Aydi, H. Lakzian, Z. D. Mitrović, and S. Radenović, "Best proximity points of MT-cyclic contractions with property UC," Numerical Functional Analysis and Optimization, vol. 41, no. 7, pp. 871-882, 2020.

[14] F. Gu and W. Shatanawi, "Some new results on common coupled fixed points of two hybrid pairs of mappings in partial metric spaces," Journal of Nonlinear Functional Analysis, Article ID 13, 2019.

[15] S. Sadiq Basha, N. Shahzad, and R. Jeyaraj, "Common best proximity points: global optimization of multi-objective functions," Applied Mathematics Letters, vol. 24, no. 6, pp. 883-886, 2011.

[16] S. Nadler, "Multi-valued contraction mappings," Pacific Journal of Mathematics, vol. 30, no. 2, pp. 475-488, 1969.

[17] H. Kaneko, "Single and multivalued contractions," Bolletino dell Unione Matematica Italiana, vol. 6, pp. 29-33, 1985. 
[18] E. Ameer, H. Aydi, M. Arshad, H. Alsamir, and M. Noorani, "Hybrid multivalued type contraction mappings in $\alpha \mathrm{k}-$ complete partial b-metric spaces and applications," Symmetry, vol. 11, no. 1, Article ID 86, 2019.

[19] S. Czerwik, "Nonlinear set-valued contraction mappings in $b$ metric spaces," Atti del Seminario Matematico e Fisico dell'Universita di Modena, vol. 46, no. 2, pp. 263-276, 1998.

[20] P. Patle, D. Patel, H. Aydi, and S. Radenović, "On $\mathrm{H}^{+}$Type multivalued contractions and applications in symmetric and probabilistic spaces," Mathematics, vol. 7, no. 2, Article ID 144, 2019.

[21] S. Basha and P. Veeramani, "Best approximations and best proximity pairs," Acta Scientiarum Mathematicarum, vol. 63, pp. 289-300, 1997.

[22] S. Sadiq Basha and P. Veeramani, "Best proximity pair theorems for multifunctions with open fibres," Journal of Approximation Theory, vol. 103, no. 1, pp. 119-129, 2000.

[23] N. Mlaiki, H. Aydi, N. Souayah, and T. Abdeljawad, "Controlled metric type spaces and the related contraction principle," Mathematics, vol. 6, no. 10, Article ID 194, 2018.

[24] M. Jleli and B. Samet, "Best proximity points for $\alpha-\psi$-proximal contractive type mappings and applications," Bulletin des Sciences Mathematiques, vol. 137, no. 8, pp. 977-995, 2013.

[25] T. R. Rockafellar and R. J. V. Wets, Variational Analysis, Springer, Berlin, Germany, 2005.

[26] N. Alamgir, Q. Kiran, H. Isik, and H. Aydi, "Fixed point results via a Hausdorff controlled type metric," Advances in Difference Equations, vol. 24, pp. 1-20, 2020.

[27] M. Jleli, B. Samet, C. Vetro, and F. Vetro, "Fixed points for multivalued mappings in $b$-metric spaces," Abstract and Applied Analysis, vol. 2015, Article ID 718074, 7 pages, 2015. 